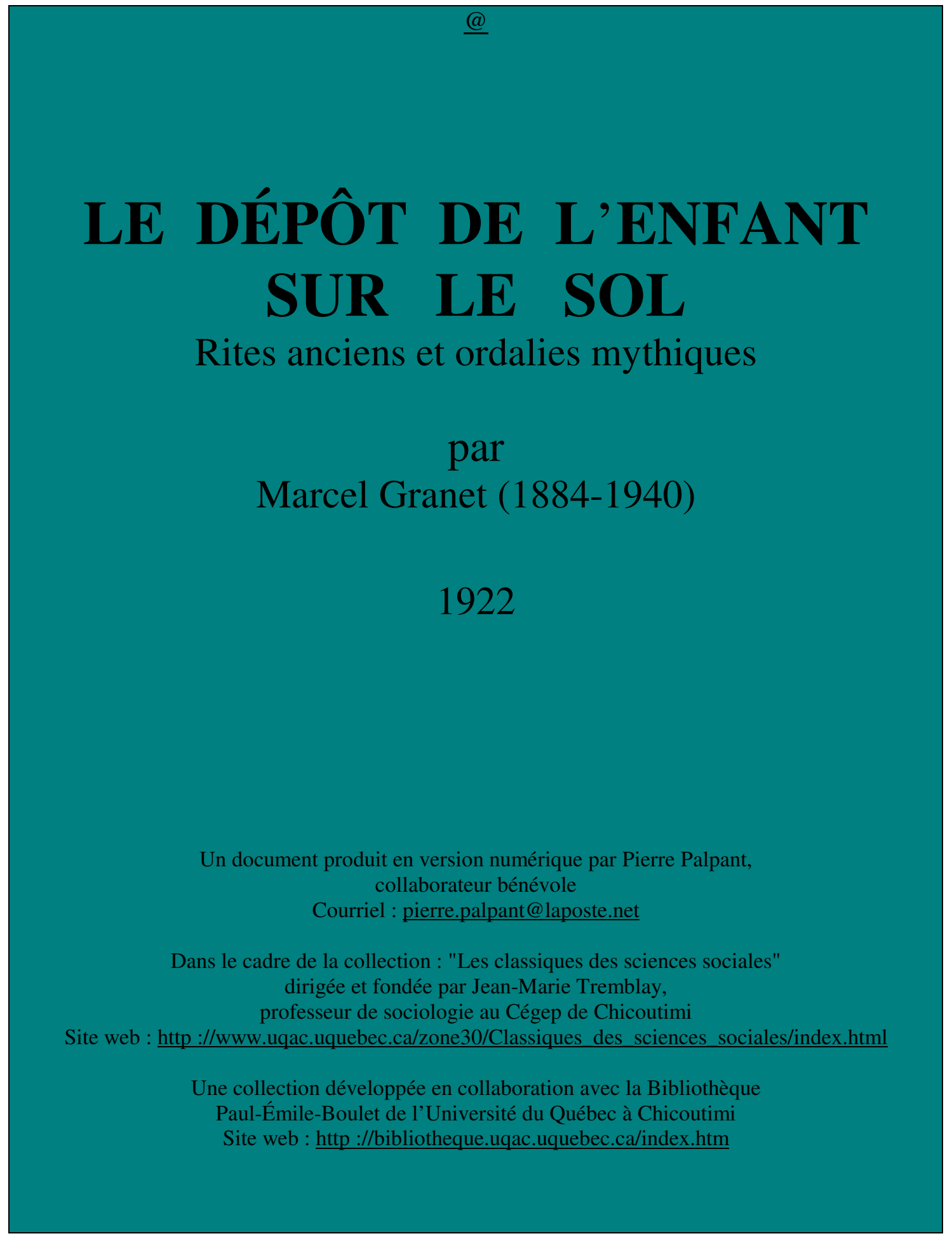


Un document produit en version numérique par Pierre Palpant, collaborateur bénévole, Courriel : pierre.palpant@laposte.net

à partir de :

\section{Le dépôt de l'enfant sur le sol}

Rites anciens et ordalies mythiques, par Marcel GRANET (1884-1940)

Article écrit en juin 1920 et paru dans la Revue Archéologique, 1922. T. XIV, $5^{\mathrm{e}}$ série. Textes rassemblés sous le titre «Essais sociologiques sur la Chine», Les Presses universitaires de France, $2^{\mathrm{e}}$ édition, Paris, avril 1990.

Polices de caractères utilisée : Times, 10 et 12 points.

Mise en page sur papier format LETTRE ( US letter ), 8.5” x 11".

Édition complétée le 30 novembre 2004 à Chicoutimi, Québec. 


\section{TABLE}

Cette table n'est pas dans l'article de M. Granet, et ne fait que rappeler pour la commodité les trois sections de l'article faites par l'auteur, avec le début de chaque section.

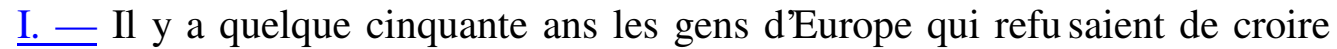
que les Chinois noyaient uniformément leurs petites filles ...

II.- Sur le même thème rituel du dépôt de l'enfant sur le sol, la pensée métaphorique a encore travaillé....

III. - Ordalie mythique ou métaphore d'édification, l'usage d'expo ser l'enfant ne s'est conservé dans la littérature ... 
Soyez bien aises quand vous trouvez l'occasion de faire quelques ouvrages un peu grossiers; cela vous fortifie et vous est très bon. Vous savez que le Saint Esprit loue la femme forte de ce qu'elle a roidi ses bras pour le travail.

Mme DE MAINTENON ( 1 ).

Il y a quelque cinquante ans les gens d'Europe qui refu saient de croire que les Chinois noyaient uniformément leurs petites filles, n'entendaient point sans chagrin raconter qu'en tout cas les malheureuses étaient toutes, à leur naissance, abandonnées, sans aucun soin, pendant trois jours, sur un tas de chiffons. Douloureuse aggravation de la diète des nouveau-nés! A dire le vrai, ces récits attendrissants s'appuyaient sur une tradition populaire; mais celle-ci, pour les temps modernes au moins, correspondait plutôt à une métaphore qu'à une pra tique effective. La métaphore cependant n'était point de pure invention, et voilà le curieux : d'un usage antique, qui n’impli quait, à l'égard des fillettes, aucune malveillance barbare, le goût du symbolisme moral a fait sortir une formule qui, peut-être, au cours des siècles, servit de justification à des traitements peu humains et qui, pour finir, a permis de jeter quelque discrédit sur les mœurs chinoises.

L'auteur de la métaphore malfais ante, on peut le deviner, est une femme de lettres ; c'est l'une des plus anciennes et des plus célèbres de la Chine, la Ts'ao Ta-kou (2) : instruite des lettres antiques, sœur d'historien, historienne, un peu pédante, elle exerça avec succès, dans le gynécée des Han, les fonctions délicates de professeur d'arts d'agrément et de maîtresse de morale. Elle écrivit, tout comme une autre, un traité de l'édu cation des filles; voici comme il débute : «Dans l'antiquité, quand il naissai t une fille, pendant trois jours on la couchait au bas du lit; on lui donnait pour hochet une (fuserole de) terre cuite ; puis, après un jeûne purificatoire, on annonçait (la naissance aux Ancêtres). On la couchait au bas du lit, pour manifester sa condition humble : son rôle était d'être (tenue) plus bas qu'un homme. On lui donnait pour hochet une (fuserole de) terre cuite pour manifester le zèle (qu'elle devrait montrer) pour le travail : son rôle était de tenir en main un (instrument de) travail. Après un jeûne purificatoire, on annonçait (la naissance) aux Ancêtres défunts, pour manifester qu'elle devait avoir pour rôle (d'assurer) la perpétuation des sacrifices. Dans ces trois choses (il faut voir) sans doute les principes fondamentaux de conduite du sexe féminin. » Ainsi étaient averties les femmes impériales d'avoir à être modestes, travailleuses et prolifiques.

La Ts'ao Ta-kou, historienne, aimait les textes qu'on cite en les commentant. Quelle est sa source ? C'est un morceau du Che king, la dernière strophe d'un hymne commémoratif qui passe pour célébrer la construction d'un palais édifié par le roi Siuan (828-782 avant J.-C.) de la dynastie Tcheou. 
Le roi y vivra heureusement; sa famille prospérera : il rêvera d'ours ou de serpents, présages de la naissance de garçons ou de filles (ㄱ)

- Or donc, il est né un garçon!

Çà ! couchez-le dessus le lit !

Çà ! vêtez-le d'habits de jour !

Çà ! pour hochet, un sceptre en jade !

Qu’il vagisse ! oh ! qu’il peut crier !

Rouges, brillent ses genouillères !

Maison royale ! ou fief princier!

Or donc, il est né une fille!

Çà, couchez-la dessus la terre!

Çà, vêtez-la d'habits de nuit !

Çà, pour hochet, une fuserole !

Rien pour le Mal! Rien pour l'Honneur !

Qu'elle borne aux repas sa tâche !

Point n'aient, ses parents, de chagrin (4) !

On entend assez, dans ces deux strophes, la joie des heureuses naissances ; il est clair que cette joie est plus franche quand il s'agit d'un garçon. Un garçon ne quitte point sa famille ; son avenir y est assuré : soit qu'il perpétue la lignée royale, soit qu'il reçoive un apanage princier, on est sûr qu'on verra briller sur lui les genouillères rouges qui distinguent les seigneurs; dès ses premiers vagissements, on reconnaît avec orgueil, à la sonorité de sa voix, la force d'âme qui fait le souverain. Il y a plus d'incer titude dans le destin d'une fille : elle abandonnera les siens pour épouser un étranger; sans doute elle aura sa part de gloire quand, aux côtés de son mari, elle participera au culte de sa nouvelle famille (5); mais enfin, elle sera subordonnée, occupée à surveiller la préparation des repas sacrificiels (ㅁ) ; elle n'agira jamais de sa propre initiative, et, qu'elle fasse bien ou mal, c'est à l'influence souveraine (7) de son seigneur que seront rapportés tous ses actes ; s'il y a de l'honneur, il sera tout pour lui. Sans doute, aussi, elle représentera, dans une cour étrangère, la politique et les intérêts traditionnels de sa propre famille ; mais quelle honte si elle ne réussit point et se voit répudiée par un mari qui déclarera «ne pouvoir assurer avec elle le service des Autels du Sol et des Moissons et du Temple ancestral » (ㅁ) ! C'est là un chagrin que ses parents ne sont point sûrs d'éviter et la pers pective des vendettas féodales (9) rend plus mélangée la joie que donne sa naissance.

L'institutrice du gynécée des Han vivait à une époque où, du moins en droit, le rôle des femmes était moins important qu'aux temps féodaux ; elle avait pour mission d'apprendre la modestie au personnel d'un harem nombreux, d'un harem où l'ordre n'était plus assuré par la pratique de la polygynie sororale (10) : en démarquant le Che king, elle a mis l'accent sur ce qui, dans le texte, pouvait manifester l'infériorité féminine. 
A l'occasion d'une naissance, les Chinois pratiquaient un certain nombre de rites qui, d'abord, semblent avoir pour fin d'orienter l'enfant vers la destinée particulière à son sexe, en lui conférant des aptitudes appropriées. Ainsi les rituels nous apprennent que, dès qu'un fils naissait (11), on disposait un arc à gauche de la grande porte; pour une fille, c'était, à droite de la porte, une serviette : il s'agit là d'attributs propres à chaque sexe. La serviette que la mère, au départ de la pompe nuptiale, avec de solennelles adjurations, attache à la ceinture de la mariée, celle-ci l'utilise pendant la nuit des noces (12). Le tir à l'arc est la grande affaire du noble : c'est en s'y montrant adroit, aux concours périodiques, qu'un vassal prouve sa droiture et mérite son fief (13). Arc ou serviette suspendus sont des souhaits contraignants, des présages efficaces de bon succès pour l'homme noble ou la future épouse.

Le Che king, en des vers d'une symétrie rigoureuse, nous fait connaître d'autres rites. On revêtait l'enfant mâle de vêtements bons pour un homme, destiné à passer ses journées hors de la maison, l'activité masculine étant d'ordre social. La fille était habillée d'un vêtement de nuit, en signe qu'elle ne déploierait d'activité qu'à l'intérieur du gynécée et pour remplir ses devoirs d'épouse. De même, pour hochet, un garçon receva it un sceptre de jade et une fille un instrument servant au tissage et fait de terre cuite (une fuserole, sans doute). Enfin, le fils était placé sur le lit, la fille à terre.

La Ts'ao Ta-kou, parmi ces pratiques, a choisi celles qui fournissaient le thème d'une allégorie profitable à son couvre d'édification. Elle n'a point glosé sur les différences d'habits : peut-être paraissait-il inutile, à ce professeur de morale des femmes impériales, d'insister sur des coutumes qui évoquent trop d'images sexuelles. Plus vraisemblablement encore ne trouvaitelle point de leçons à tirer d'un usage qui (comme le rite de la serviette et de l'arc) montre simplement dans quels milieux différents se situent l'activité masculine ou féminine. Surtout, le texte où elle s'appu yait ne lui fournissait, sur ce point, aucun élément de développement métaphorique. Il en était autrement des vers :

Çà ! couchez-le dessus le lit !

Çà ! couchez-la dessus la terre !

Çà ! pour hochet, un sceptre en jade !

Çà ! pour hochet, une fuserole !

Rien qu'à évoquer les deux enfants, tenant en main, l'un une tablette de beau jade, emblème de haute dignité, l'autre un humble instrument de travail fait de terre vulgaire, on a moyen d'inculquer aux femmes des sentiments de modestie. Non point sans forcer le sens du texte qu'on utilise, ni sans fausser la valeur de l'usage qu'il décrit. Donner à un garçon pour hochet une tablette d'investiture, comme suspendre un arc à la porte, donner à une petite fille une fuserole, de même que suspendre une serviette, c'est simplement vouloir les prédisposer, l'un à remplir les devoirs qui lui incomberont dans la hiérarchie féodale où il prendra place, l'autre à tenir dans la maison les fonctions que les 
rites assignent à l'épouse. Que, portant à la ceinture la serviet te rituelle, la femme accomplisse ses devoirs conjugaux (14), ou que, fuserole en main, elle préside à la confection des vêtements sacrés (15), son activité, pour être différente, n'est ni moins impor tante, ni moins noble que celle du mari quand il participe aux cérémonies de la cour seigneuriale. Mais le texte prêtait à la glose - à condition d'insister sur la matière dont étaient faits les deux hochets : la Ts'ao-Ta-kou a construit cette image tendancieuse de deux enfants s'amusant avec des jouets de valeur bien inégale : présages, pour le garçon, d'une noble vie qui s'écoule doucement, occupée par de belles cérémonies, et, pour la fille, d'une existence employée à de dures et grossières besognes.

L'esp rit qui a conduit ce développement métaphorique est plus sensible encore pour l'autre rite. Le Che king dit que le nouveau-né est couché sur le lit et la nouveau-née à terre; il a suffi à la $T s^{\prime}$ ao Ta-kou de condenser les deux vers en une phrase et d'écrire que l'on couchait la fille au bas du lit, pour obtenir aussitôt une image éclatante de l'infériorité féminine. Comme elle savait manier sa langue, en bonne Chinoise, elle a réussi à employer le même mot pour définir cette infériorité juridique et pour peindre l'image de la fillette déposée sur le sol. Par cet artifice ingénieux, elle a fixé pour l'avenir le sens des vers qu'elle commentait et de l'usage qu'ils notaient ; du même coup, en lui donnant une valeur nouvelle, elle a aidé à se maintenir une pratique dont elle et ses contemporains ne sentaient plus la signification originale. Le traité de morale d'un auteur aussi subtil méritait le succès ; il l'a eu : sans doute, sous son influence, beaucoup de gens bien pensants, pour inculquer tôt la modestie à leurs filles, ont voulu, à leur naissance, les laisser à terre pendant trois jours.

Pendant trois jours, le Che king ne le disait pas et, en le disant, la $T s^{\prime}$ ao Ta-kou a rendu (du moins aux archéologues) un grand service. Dans les usages modernes, c'est le troisième jour après la naissance que se fait une cérémonie de purification, le lavage du nouveau-né ; dans l'antiquité, c'était le troisième jour (16) que l'on commençait à porter l'enfant.

Ce jour-là, le père, averti de la naissance, ordonnait un sacrifice, par lequel, comme l'indique la Ts'ao Ta-kou, étaient, à leur tour, avertis les ancêtres. Ainsi le nouveau-né était reçu dans la famille. Un vassal, désigné par la consultation .des sorts, se purifiait, revêtait des habits de cérémonie, et, pour la première fois, portait l'enfant dans les bras. Le service quil rendait ainsi était assez méritoire pour qu'on le payât du présent rituel de quelques pièces de soie. Le nouveau-né, alors seulement, était remis à sa nourrice, c'e st-à-dire à sa mère pour les gens du peuple et les nobles du dernier rang, et, pour les grands-officiers et les seigneurs, à une mère nourricière dirigée par une gouvernante et assistée par une gardienne, désignées, toutes trois, par les sorts (17). - Ces gardes le tenaient enfermé dans une pièce réservée où nul n'entrait sans raison grave (18) (car l'esprit vital et le souffle d'un enfant sont 
sans force) (19), jusqu'à la fin du troisième mois. Ce temps de retraite terminé, il était présenté en cérémonie à son père, à son grand-père et recevait un nom personnel dont on faisait part à la parenté et qu'on enregistrait à l'état civil (20). Il continuait jusqu’à la troisième année, à vivre avec sa nourrice ; sa nourriture terminée (21), il prenait enfin part, avec les autres enfants, à la vie commune du gynécée.

La cérémonie du troisième jour, comme celle du troisième mois, clôt une période de la vie enfantine et ouvre une période nouvelle: ces périodes semblent correspondre à des stages que ferait le nouveau-né avant de pénétrer dans la vie familiale. L'isolement, qu'on lui impo sait pendant ces retraites successives, presque absolu, tant qu'il n'avait point de nom, était total les premiers jours, où on l'abandonnait, garçon sur le lit ou fille sur la terre, sans vouloir encore le nourrir. Pourquoi donc cet abandon et cette diète?

- La gardienne de l'enfant, si c'était un garçon, ne le recevait pas des mains du vassal chargé de le porter pour la première fois, avant que l'on n'eût tiré six flèches à l'aide de l'arc sus pendu trois jours à la porte (22). Cet arc n'était point un arc ordi naire ; il devait être fait en bois de mûrier et les flèches étaient d'armoise : le mûrier est l'un des arbres sacrés (23), l'armoise une plante salutaire, efficace contre les souillures (24). On tirait les flèches vers le ciel, la terre et les quatre points cardinaux ; un commentateur pense que c'était là un rite d'élimination des Malheurs qui peuvent venir de toutes les directions (25); un autre indique quil s'agit d'un usage antique (26) ; divers textes montrent que les anciens Chinois espéraient en tirant, avec des arcs faits d'un bois sacré, des flèches d'armoise, de r oseau ou d'épine, éliminer magiquement les Calamités, le Mal, le Néfaste (27).

Les rituels s'occupent moins volontiers des filles que des garçons ; pour elles, disent-ils, on ne tirait point de l'arc : en effet, puisqu'à leur nais sance ce n'était point un arc que l'on suspendait à la porte. On y suspendait une serviette : on ne nous dit pas si, le troisième jour, on s'en servait et de quelle façon. Sans doute était-elle bonne à évacuer des souillures, cette serviette que les femmes doivent toujours porter à la ceinture à côté d'un sachet de parfum et dont il est dit que, la nuit des noces, elle servait à purifier (28).

- Les pertes sanglantes (29) de la naissance souillent la mère, et l'enfant participe à cette impureté : il faut bien l'en purger avant de le nourrir. Mais les rites du troisième jour et la période d'isolement qu'ils couronnent $\mathrm{n}$ 'ont-ils qu'une fin négative ?

Les chroniques (30) nous ont conservé la mémoire d'un antique seigneur du pays de Tch'ou qui, pour s'acquitter de ses devoirs de roi, n'avait qu'un arc de pêcher et des flèches d'épine : avec ces armes magiques, il écartait de son pays les Calamités ; avec elles encore - car le texte met leur possession en parallèle avec celle des talismans qui fondent les pouvoirs seigneuriaux -, il exerçait positivement sa puissance ; elles lui permettaient de faire sentir son 
action dans tous les coins de son domaine. Il y a, de même, des textes et des commentateurs pour nous dire qu'en tirant de l'arc pour un nouveau -né, on établissait une liaison entre lui et les lieux où, plus tard, devrait s'exercer son action.

«Il fallait que, d'abord, il eût des intentions sur les lieux où il agirait, pour qu'ensuite il pût manger des grains (31).

Manger des grains (recevoir sa part des offrandes données au seigneur en hommage), c'est avoir son rang marqué dans la hiérarchie féodale, où, précisément, chacun se classe d'après son habileté au tir de l'arc (32). Tant qu'on n'a pas tiré de l'arc en sa faveur, un garçon ne doit point manger : il doit attendre que, par ce moyen, on le mette en communication avec le sol nourricier du pays qu'il gouvernera ou aidera à administrer.

- Un rituel développé (33), à l'usage de l'héritier présomptif du royaume, montre cette idée bien au clair. Pour une telle naissance, on se sert de cinq arcs de bois différents, choisis chacun pour correspondre aux quatre Orients et au Centre; on dispose de cinq flèches pour chaque arc; on en tire trois seulement, et, naturellement, dans la direction de l'espace dont est l'emblème le bois de l'arc que l'on emploie ; les deux flèches restantes de chaque lot sont, de même, suspendues à la gauche des quatre Portes (Est, Ouest, Sud, Nord) de la capitale et celles de l'arc de mûrier (qui correspond au Centre) à la gauche de la Porte d'enceinte des Autels du Sol et des Moissons. Flèches jetées à toutes les directions de l'espace, flè ches offertes aux points où culmine l'énergie sacrée de la Terre natale, procédé d'évacuation, procédé d'ap proche, élimination, acquisition, tout cela se voit bien dans ce texte, fruit d'une savante technique rituelle, et fait clairement apparaître le caractère double de la cérémonie du troisième jour.

Ce texte a encore le mérite de faire sentir que l'élimination de souillures et l'acquisition de puissance positive qui donnent au nouveau-né le droit d'être nourri s'obtiennent, toutes deux et conjointement, par un procédé à la fois énergique et prudent de mise en contact avec la Terre nourricière. Or, si tel est bien le caractère de la cérémonie qui clôt les trois premières journées de la vie enfantine, n'est-il pas remarquable que, pendant ces trois jours où on ne le porte point encore, l'enfant ait été aban donné (comme la Ts'ao Ta-kou, le Che king nous l'apprennent) garçon, sur le lit et, fille, sur la terre ?

Le contact, ni pour l'un ni pour l'autre, n'est immédiat ou trop intime, car, tous deux sont vêtus; mais il se fait de manière différente. La fille est déposée, tout simplement, sur le sol ; en celui-ci, dans les trois jours, va se disperser la souillure, cependant que la faible vitalité de l'enfant sera réconfortée par l'énergie terrestre. Mais, pour le garçon, il faut autre chose que de le déposer en un point quelconque du sol ; car lui, le mariage ne doit point le dépayser et il passera tous ses jours dans la maison paternelle : on le couche sur le lit. • Or, si dans les domaines d'archi tecture compliquée où vivait la Noblesse, la mère pour accoucher se retirait dans une chambre 
latérale, dans la maison antique qu'habitaient toujours les gens du peuple, le mari cédait, pour le temps des couches (느), sa place à la femme, et le lit, sur lequel on déposait le garçon, se trouvait certainement, au moins en ce cas, dans la partie sacrée de la maison, dans l'angle Sud-Ouest (35), dans l'endroit le moins éclairé, là où l'on conservait les semences (36) et où l'on faisait les offrandes réservées à ceux qui n'étaient point morts sans postérité (37), dans le coin, enfin, qui appartenait en propre au chef de famille (38) parce qu'on le considérait comme la résidence des puissances tutélaires de la famille et de la maison. Là, pendant ses trois jours d'abandon, le petit garçon peut recueillir les forces particulières qui émanent du sol familial.

Mais le génie spécifique qu’il assimile n'est qu'une forme particularisée de l'énergie que dégage la terre et qui donne un ton plus haut à la vitalité de la petite fille. L'esprit est le même dans toutes les pratiques qui distinguent garçons et filles : celles-ci ne sont point laissées à terre par mépris, mais parce qu'elles ont à incorporer en elles la bonne influence de la. large Terre où vivent, à distance de leurs parents, leurs maris futurs (39) ; ceux-là sont placés sur le lit, non pas spécialement pour leur faire honneur, mais pour les orienter vers leur destinée de chefs de la famille natale et de maîtres du Sol domestique. Dépôt sur le lit, dépôt sur le sol ne sont que deux aspects de la présentation de l'enfa nt à la Terre natale.

D'ailleurs, le garçon n'est pas uniquement soumis à l'influence spécifique du Sol patrimonial ; un texte l'affirme de façon formelle (느): tous les enfants, garçons comme filles, étaient, aux premiers moments de la naissance, placés sur la terre. Et le même texte, rapprochement significatif, signale que le dépôt sur le sol, obligatoire pour tous les nouveau-nés des deux sexes, l'est aussi pour tous les mourants ; " on enlevait, dit-il, le moribond du lit (pour le déposer à terre) dans l'espoir que le souffle de vie lui reviendrait (ㄴ1). » Une même confiance dans l'action vivifiante de la Terre natale explique ces deux rites symétriques de la naissance et de la mort.

- Une telle pensée n'est point surprenante à trouver chez les anciens Chinois dont le sentiment le plus fort était celui d'autoch tonie et le culte le plus ancien celui qu'ils rendaient à des Centres Ancestraux ou, mieux, aux Lieux-Saints d'une race et d'un pays, dispensateurs de la fécondité des familles et des années (42). Dans le culte féodal, aux manifestations localisées de la puissance terrestre correspondit la représentation de génies du Sol, organisés hiérarchiquement, tels des seigneurs et des vassaux, tous conçus comme des Héros et pourvus d'une apparence mas culine (43). Lorsque l'Empire fut formé et l'unité nationale mieux sentie, un culte de la Souveraine Terre se fonda, où l'on retrouva, sublimée, l'idée d'une puissance maternelle, nourricière, prochaine et d'aspect multiple : tout opposée à celle du Ciel, représenté comme un père et un chef, unique, lointain, dominateur, sévère. Le culte de la Terre attendit pour s'organiser face à celui du Ciel que s'établît l'Unité impériale ; mais, depuis bien des années, l'idée de la Terre Souveraine s'opposait, dans la pensée religieuse, à celle du Ciel Majestueux, 
respectivement définies par les attributs propres à la puissance paternelle et à la bienveillance maternelle (4ㅜ).

Le garçon, après avoir, dans un premier contact avec la Terre-mère, réconforté son énergie vitale, demeure jusqu'au troisième jour sur le lit paternel : dans le coin le plus sacré de la maison de famille, il acquiert, avec les vertus particulières au Sol natal, la puissance virile qui l'habilitera à rendre le culte du génie du lieu, et à posséder en maître la terre domestique. Cette puissance, une fille n'a point à l'acquérir, mais il convient qu'elle s e pénètre plus généralement et plus profondément des vertus nourricières de la Terre-mère : donc, abandonnée les trois premiers jours, tout entiers, sur la terre, elle en tire, avec une vie plus riche, la confirmation de ses attributs féminins.

Si le stage de trois jours sur le sol nourricier, condition préalable au commencement de la nourriture, sert encore, comme la cérémonie du troisième jour, à éliminer les souillures de la naissance qui rendent si délicat le contact de l'enfant et font reculer si lo in le jour où on peut le prendre dans les bras, un autre motif se voit à la différence de traitement qui s'établit entre les garçons et les filles. Quand, pour ceux-là, l'avènement de la puissance paternelle rendit nécessaire l'emploi d'un rite d'approche du lit domestique, l'organisation de la famille impliqua, pour les filles, une certaine exclusion de la vie religieuse nouvellement orientée. Destinées à une existence recluse (45), elles semblèrent chargées de qualités qui commandaient l'isolement : il y a des chances que le rite du dépôt sur le sol, conservé pour elles sous sa forme originale, apparût surtout avec sa valeur négative et comme s’il était uniquement imposé par la nécessité de chasser une souillure.

Et si, comme l'a rc symétrique, la serviette, exposée au côté droit de la porte, servait, comme on doit le supposer, à une espèce de lustration, on peut se demander si l'usage qu'on en faisait n'est point à l'origine de la tradition populaire qui veut que l'on abandonne les filles sur un tas de chiffons. Dans la vallée de Nguon-son (46), le nouveau-né est déposé sur quelques habits placés sur un tamis ; dans la Chine moderne (47), celui qui habille à neuf un mort interpose un van entre le sol et ses pieds. Entre la fillette chargée d'impuretés et la Terre-mère où celles-ci vont se diffuser, il ne convenait point sans doute d'établir le contact sans précaution. L'officier chargé de disperser avec ses flèches, aux quatre coins de l'espace, l'impureté de la naissance, doit se purifier, jeûner et ne peut se servir que d'un arc de bois consacré. La désacralisation de l'enfant, qui aboutit à une sacralisation nouvelle, n'exige pas seulement du temps, mais des formes prudentes. Dès qu'on eut reconnu, en une fille, une plus grande somme d'impuretés, il s'imposait et de l'abandonner plus long temps sur la terre et de ne point l'y laisser en contact trop intime.

Ainsi le rite originel du dépôt sur le sol s'est maintenu pour les filles, mais il a changé de valeur. Le changement s'est fait, tout naturellement, en fonction 
de transformations sociales : il est remarquable qu'une étape importante de l'évolution et, peut-être même, le maintien de l'usage soient dus au travail de réflexion morale et de pensée métaphorique que le texte de la $\mathrm{Ts}^{\prime}$ ao $\mathrm{Ta}-\mathrm{Kou}$ nous a permis d'analyser. 
- Sur le même thème rituel du dépôt de l'enfant sur le sol, la pensée métaphorique a encore travaillé, mais d'autre manière soutenue, cette fois, par l'esprit d'invention historique, par l'ima gination généalogique, elle a abouti à la création d'une légen de poétique : la Nativité de Heou-tsi.

- - Heou-tsi, fils de Ti Kou (Kao-sin), fils de Kiao-ki, fils de Hiuan-hiao, fils de Houang-ti, est le grand Ancêtre de la maison royale des Tcheou (48). Descendant de Houang-ti à la cinquième génération, il fonda une branche familiale distincte (nom $K i$ ) et, par lui, les Tcheou, qui en furent le principal rameau, se rattachent à la première grande lignée. De même, s'y rattachaient les Yin, prédécesseurs des Tcheou, par l'intermédiaire de Sie, fondateur de la famille Tseu (무).

Rattachés à Houang-ti, il importait que les Tcheou (comme les Yin) le fussent, car Houang-ti est le grand Ancêtre Civilisateur.

«Grâce à lui, les dix mille pays vécurent en harmonie... Il planta aux époques voulues les cent espèces de céréales, d'arbres et de plantes; il favorisa le développement des oiseaux, des quadrupèdes, des insectes et des reptiles ( $\underline{50})$.

«Sa Vertu eut une influence bienfaisante et profonde sur sa postérité : c'est pourquoi ses descendants furent tous promus à leur tour au rang de Fils du Ciel. C'est ainsi que le Ciel récompense celui qui possède la Vertu (51).

$Y u$, qui descendait, lui aussi, de Houang-ti à la cinquième génération (52) et qui fonda une branche familiale nouvelle (nom Sseu) fut le premier souverain de la première maison royale de Chine, celle des Hia. Possesseur de tout l'empire, il eut, comme Houang-ti, une vertu complète.

«Sa voix était l'étalon des sons; son corps était l'étalon des mesures de longueur ( $\underline{53}$ ).

Ni Sie, ni Heou-tsi, ses contemporains, ne pouvaient être investis d'une Vertu illimitée; ils durent (comme Yu avant son avènement) se contenter d'une province de la Nature. Sie eut en charge le département de l'instruction, et Heou-tsi celui de l'agriculture (54), d'où son appellation (55). Heou-tsi veut dire : Prince Tsi (millet) ou, si l'on veut : Prince des Moissons, le millet, Tsi, étant la principale des céréales et le fond de l'alimentation ancienne ( $\underline{56}$ ). Par l'exerc ice de sa Vertu ainsi spécialisée, « les cent céréales furent abondantes en la saison voulue »( $\underline{57})$. Il obtint donc d'être associé au culte royal rendu sur les Autels du Sol et des Moissons (= Tsi, millet) (ㅁ8). 
Ayant ainsi rénové par une activité méritoire la Vertu héritée de Houang-ti, fondateur de la première race, Heou-tsi avait de quoi devenir le premier Ancêtre d'une dynastie nouvelle, issue de la branche familiale détachée (59) avec lui, cinquième descendant du premier Héros Civilisateur, de la grande souche initiale. Sa tablette fut honorée à perpétuité dans une salle placée au fond du Temple Ancestral de la famille $K i$, dont les souverains Tcheou représentaient la lignée principale.

Heou-tsi occupait la place d'honneur à la fois dans le culte du Temple Ancestral et dans celui des Autels du Sol et des Moissons, cultes dont la dynastie des Tcheou (comme toutes les autres) tirait la puissance qui autorise à gouverner l'Empire. Les sacrifices magnifiques de ce double culte s'accompagnaient de chants rituels. La mémoire de Heou-tsi fut célébrée par les Hymnes que les poètes composèrent à la gloire de la lignée des Fils du Ciel (무).

Fils du Ciel, tel est le nom superbe que l'imagination religieuse et la poésie dynastique donnèrent au Souverain : chargé d'assurer l'ordre et la prospérité de la Terre chinoise, il avait pour office de visiter, à la saison qui convenait, les seigneuries des régions de l'Espace et de promulguer les règlements saisonniers, grâce auxquels le travail des hommes peut se conformer heureusement au cours du Temps. Maître du Calendrier et dispensateur d'une Influence qui, comme celle du Ciel, s'exerçait en temps voulu au point marqué, le chef de l'Empire, le maître unique de la Terre étendue sous le Ciel, apparaissait comme le délégué terrestre d'une Puissance régulatrice céleste qui l'investissait du mandat de la suppléer, comme fait, à la mort du père, le fils. Un hymne qui nous dit : «Quelle lumière ! quel éclat ! a la maison des Tcheou ! » débute par ces vers : "Au temps voulu, je visite les principautés : l'Auguste Ciel, voici qu’il me traite comme son fils (61)!»

Dès que l'on imagina avec cette force le rapport unissant le Souverain au Ciel, dès que, se développant avec la science du Calendrier, et croissant de pair avec le sentiment de l'unité nationale, l'idée s'éleva d'une Puissance régulatrice, céleste, souveraine, unique, et qu'il s'établit un cu lte astronomique réservé à la Cour royale et rendu au Souverain d'En-haut Auguste Ciel (62), il s'ouvrit un champ nouveau pour la poésie dynastique et l'invention généalogique.

De même qu'il paraissait nécessaire, pour sacrifier, sur l'Autel du Sol et des Moissons, à des Forces agraires bienfaisantes qu'on ne personnifiait point, de leur associer un Ancêtre héroïque, Médiateur humain, de même l'on associa au Ciel, puissance mal individualisée et lointain auteur de la souveraineté dynastique, l'Aïeul le plus reculé de la maison régnante (63) : de celui-ci la poésie fit un véritable fils du Ciel. Par lui la race royale ne remonta plus seulement au grand Héros civilisateur, souche originelle de toutes les lignées, mais encore au Principe, nouvellement entrevu, de tout ordre et de toute loi. 
- Il y avait, dans les généalogies des diverses familles royales, un point où leur orgueil pouvait souffrir: toutes savaient montrer comment elles remontaient au premier Héros; mais elles devaient bien reconnaître que l'aïeul, dont elles se réclamaient pour établir cette descendance, avait été écarté du pouvoir suprême et réduit à fonder une lignée secondaire. Sans doute, elles pensaient réparer cette infériorité en glorifiant les mérites acquis par lui dans la direction d'une province particulière ; sans doute aussi, elles faisaient valoir quỉl était né plus noblement que ceux qui, d'abord, l'avaient primé : les Tcheou, par exemple, affirmaient que Heou-tsi, leur auteur, était le fils de la première épouse, - Sie, son demi-frère, auteur des Yin leurs prédécesseurs, n'ayant eu pour mère qu'une femme de second rang de Ti Kou. Il restait que Heou-tsi (pas plus que Sie) n'avait pas été traité en fils de droite lignée : de ces fils secondaires, quelle gloire n'y avait-il pas à faire de vrais fils du Ciel

Sie et Heou-tsi passaient pour descendre de Houang-ti à la cinquième génération, souche comprise: dans l'organisation familiale des temps féodaux, la parenté, manifestée par le deuil, n'existe, entre collatéraux, que sills possèdent un Ancêtre commun qui reçoive encore un culte personnel, c'est-à-dire dont la tablette n'ait point été encore transportée pour rejoindre, à la masse commune, les tablettes des Ancêtres reculés (64); quand meurt le représentant de la branche aînée, la tablette de son trisaïeul est transportée et les collatéraux qui descendent de ce trisaïeul n'ont plus de parenté avec la droite lignée. Ils forment des rameaux séparés et la famille qu'ils fondent peut recevoir un nom nouveau. Ce nom, dans lequel s'exprime la Vertu caractéristique d'une famille, est considéré comme le don d'une Puissance Souveraine.

Une tradition (65) affirmait que Sie et Heou-tsi avaient relu un nom de famille - c'était déjà un sérieux titre de gloire - du grand Souverain mythique Yao. Il était, en effet, conforme au droit public que le Souverain en donnant un fief conférât un nom de famille. D'après une autre tradition, Heou-tsi, investi par Yao des fonctions de Prince des Moissons, reçut de Chouen, successeur de Yao, le fief de T'ai (66).

Or, la mère de Heou-tsi, femme de Ti Kou, était une fille de la famille seigneuriale de T'ai (67). De bonne heure, les Chinois (68) notèrent que la lettre qui, dans leur écriture, signifiait à la fois nom de famille et filiation était une combinaison du signe naître et du signe femme. Ils notèrent encore que ce signe femme entrait dans la plupart des combinaisons graphiques employées comme nom de famille (69). Ils ne tirèrent pas de ces remarques l’idée que la filiation avait pu être utérine dans les temps anciens de la Chine : elles les induisirent à penser que les Mères des Héros, chefs de grandes lignées, avaient, et non pas les pères, joué, à la naissance de leurs fils, le rôle le plus éminent. Ils contèrent que si Heou-tsi, par exemple, avait fondé la famille Ki 
et Sie la famille Tseu, c'est que leurs mères les avaient conçus l'une en marchant sur de grandes traces de pas (Tsi) et l'autre en avalant un neuf (Tseu) d'hirondelle (무).

Les légendes qui s'établirent ainsi ne sortaient point de simples jeux de mots. J'ai montré ailleurs qu'elles s'expliquent fort aisément par le souvenir des Fêtes anciennes du mariage (71) : elles ne sont qu'une transposition des rites qui se pratiquaient, à l'occasion de ces Fêtes, dans les Lieux Saints, dans les Centres Ancestraux des différents pays chinois. Une fois établies, elles fournirent des thèmes merveilleux à l'imagination mythique.

Dès que les mours nobles et l'usage des gynécées firent paraître absurde l'idée de princesses participant à des fêtes agraires, les conceptions que contaient ces légendes apparurent non comme l'effet de rites d'un usage général, mais comme des miracles réservés à l'avènement des grands hommes. Les poètes chargés de glorifier les races royales présentèrent ces conceptions comme miraculeuses, comme l'œuvre même du Ciel. « Le Ciel donna son ordre à l'hirondelle ; elle descendit et fit naître les Chang (les Yin) (72) » dit le panégyrique de la race des Yin; celui des Tcheou, pour célébrer Kiang Yuan, première aïeule de la race, nous la fait voir qui, après des lustrations et des sacrifices, a foulé la trace du gros orteil du Souverain (céleste) » (3).

«Le Souverain d'En -haut se reposa sur elle », dit de Kiang Yuan un autre hymne (74); et le terme qu'il emploie ( $\underline{75})$ indique un contact véritable : celui d'une union réelle. Il est dit encore qu'à ce contact elle éprouva comme un frémissement, analogue, ajoutent les commentaires, à celui que produit le contact viril (76). Le Ciel, d'après la glose, fit descendre en Kiang Yuan la semence de vie (77). Le Po hou t'ong (78) considère que, dans de tels cas, l'œuvre de la naissance résulte d'une distribution du Souffle (ou de l'Esprit) Céleste. Et le Chouo wen (9ㅜ) conclut :

«Les mères des Saints Héros de l'ancien temps, étant émues par le Ciel, enfantèrent des fils que, pour cela, on appela Fils du Ciel.

Lorsque, grâce à la poésie panégyrique, Heou-tsi fut devenu le fils du Ciel, il ne cessa point pour cela d'être fils de l'homme et rejeton de la grande lignée héroïque, issue du premier civilisateur ; les chroniques continuèrent d'écrire : Houang-ti engendra Hiuan-hiao; Hiuan-hiao engendra Kiao-ki; Kiao-ki engendra $\mathrm{Ti} \mathrm{Kou}$; et $\mathrm{Ti} \mathrm{Kou}$ engendra Heou-tsi. Seulement Ti Kou, père de Heou-tsi, occupa dans le culte une place un peu spéciale. - En arrière du Temple des Ancêtres où la place d'honneur était prise par la tablette de Heou-tsi, les Tcheou bâtirent une chapelle quils appelaient (0) le temple fermé. Un auteur ancien, cité par Mao Tch'ang, Mong Tchong-tseu, la désigne du nom de MEI Kong et indique qu'elle était consacrée à Kao MEI. On ne faisait point d'offrandes dans cette chapelle ; mais, à l'époque où tombaient les fêtes anciennes des fiançailles, à l'équinoxe de printemps, jour où reviennent les 
hirondelles, le chef de l'État, suivi par ses femmes, allait, dans la banlieue sud de la capitale, offrir un grand sacrifice à $\mathrm{Kao}-\mathrm{Mei}$, protecteur des mariages (81). Et l'on nous dit que KaO Mei , c'est KAO-sin, autre nom de Ti Kou mari de Kiang Yuan et père de Heou-tsi, fils du Ciel (82). Ainsi, comme de juste, dans la théologie des Tcheou, de par l'éminente dignité de son fils Heou-tsi, Ti Kou est devenu le patron de la vie conjugale.

D'après une autre tradition (3) le Temple retiré était assigné à l'ancienne aïeule Kiang Yuan: c'était un lieu de profonde retraite (84). Cette retraite profonde, par les mots dont on la qualifie, rappelle tout de suite le temps de réclusion imposé aux jeunes vierges avant le jour des noces ; elles devaient, dans les usages féodaux, accomplir, avant le mariage, une retraite de trois mois, dans un bâtiment du Temple Ancestral (도). Une retraite de même durée était aussi une obligation rituelle pour les femmes avant leurs couches (6ㅜ). Kiang Yuan, les hymnes insistent (7) sur ce point, se soumit entièrement à cette obligation avant la naissance de Heou-tsi. Les mêmes hymnes racontent qu'avant d'être visitée par le Souffle du Ciel, elle avait offert des sacrifices de lustration au Souverain d'En-haut: ils rappellent ces ouvres de purification quand ils chantent les mois de grossesse (88). C'est ainsi que la pureté de Kiang Yuan fut un thème sur lequel on insista : "Qu'elle est vénérable Kiang Yuan! Sa Vertu fut sans défaillance ! Le Souverain d'En-haut s'appuya sur elle (오)!»Ce fut son irréprochable Vertu qui parut lui avoir valu la Visitation du Ciel: elle fut glorifiée d'avoir conçu sans le secours d'un homme (90) et, en raison de ce miracle, par lequel était rendue manifeste la transcendance divine (91) qu'il y avait en elle, elle mérita d'être regardée comme une sainte, vierge et mère du fils du Ciel.

Vierge fécondée par le souffle du Ciel, son enfant ne naquit point dans les labeurs imposés aux femmes du commun: elle le mit au monde «sans rupture, sans fissure, sans mal, sans lésion » (92). Ce premier né vint au jour comme l'agneau d'une brebis: à conception sine concubitu, naissance merveilleuse.

Né dans le prodige, fruit d'une Vierge sans tache, fils du Ciel qui régit les saisons et les hommes, rejeton de la première lignée de Héros Civilisateurs, auteur d'une dynastie nouvelle qui pré tendait apporter au monde le règne de la Grande Paix (93), Heou-tsi méritait d'être, auprès du Souverain d'En -haut, le Médiateur de sa race - et voici la prière qu'on lui adressait en effet :

O Civilisateur Heou-tsi !

Puissant Associé du Ciel !

Nourris de grain notre grand peuple !

Rien n'est sans ta Sublimité !

Donne-nous le froment et l'orge !

Le Souverain veut que tous mangent

Sans borne ici, ni là limite, 
Étends ta Loi sur tout l'Empire (모) !

Mais Heou-tsi méritait encore qu'on lui prêtât une vie pleine d'œavres et une enfance miraculeuse.

«Homme fait, il se plaisait au labourage; il connaissait ce qui convient à la Terre; les céréales convenables étaient semées et donnaient des récoltes : tout le monde le prenait pour modèle (95).

L'empereur Chouen fit de lui cet éloge : "Au temps où le peuple aux cheveux noirs était affamé, vous, Heou-tsi, vous avez semé et transplanté les cent céréales (96) !»Il tenait ainsi les promesses d'une enfance riche en prodiges : grand et fort, dès ses jeunes ans, il savait à peine manger que, déjà, il cultivait heureusement la terre: "Ses grands haricots flottaient au vent comme des bannières! Ses moissons, semées en lignes, étaient très belles (97) !»

- Ces labeurs héroïques, on pouvait les présager dès ses premières heures. Écoutez plutôt l'hymne qui les chante et qui éclate (98) joyeux comme un Noël .

Or, dans la ruelle, abandonné,

Boufs et moutons l'ont protégé !

Or, au bois bas, abandonné,

Bûcherons s'y sont rencontrés

Or, sur la glace, abandonné,

Par un oiseau il fut couvé !

Et l'oiseau puis alors de fuir !

Heou-tsi puis alors de vagir !

Qu'on l'entend loin ! Qu'on l'entend bien !

Ses cris emplissent le chemin !

C'était, certes, une image bien émouvante que celle de la Nativité abandonnée du petit Heou-tsi et un admirable contraste avec ses saintes origines et la gloire promise à ce fils du Ciel. Comme pour toutes les autres légendes du cycle de Heou-tsi, il n'est g uère possible d'y voir autre chose que le souvenir de rites anciens magnifiés par la poésie panégyrique; on y retrouve, à l'occasion d'un Héros et sublimée par le fait même, la pratique de l'abandon de l'enfant sur le sol.

- Nous connaissons, par un texte assez récent, un cas où le père refuse de relever l'enfant déposé sur le sol, parce que, né dans un jour néfaste - le 5 du cinquième mois - on peut prévoir pour lui une destinée malfaisante (99). Entre le rite du dépôt et l'exposition définitive, la distance n'est pas très grande. - Un cas célèbre d'exposition est celui de Pao Sseu (100), femme funeste qui perdit le roi Yeou, qui faillit ruiner la dynastie des Tcheou, et qui, dès sa naissance, était chargée d'une irrésistible puis sance néfaste. Elle était née, elle aussi, d'une vierge-mère, mais dans dhorribles conditions 
maléficientes. Dans les temps anciens, deux dragons s'étaient présentés à un souverain Hia comme d'anciens princes de $\mathrm{Pao}$; ils ne voulurent se retirer qu'après avoir laissé de leur écume sur une pièce d'étoffe que l'on garda, cachée dans un coffret. Longtemps après, un prédécesseur du roi Yeou eut l'imprudence d'ouvrir le coffret ; l'écume se répandit dans le palais ; comme on ne pouvait l'enlever, le roi fit venir des femmes toutes nues pour vociférer contre elle; elle se transforma en reptile, pénétra dans le gynécée et vint féconder une petite fille de sept ans. A quinze ans, celle-ci mit au monde une fille ; «sans avoir eu de mari, elle enfanta ; saisie de crainte, elle exposa son enfant». Or, en ce temps-là, Siuan, le roi alors régnant, effrayé par une prédiction, avait donné l'ordre d'arrêt er, pour les mettre à mort, un homme et une femme détenteurs d'un arc de mûrier dont parlait cette prophétie inquiétante ; ce furent eux précisément qui, fuyant la colère du roi, trouvèrent la petite fille gisant sur un sentier et criant. Ils l'emmenèrent dans leur fuite à $P a o$ (naturellement) où elle grandit et d'où elle revint, sous le nom de Pao $\mathrm{Sseu}$, afin d'être, pour son malheur, la favo rite du roi Yeou.

Pao Sseu avait sa mission à remplir ; l'abandon ne put rien contre elle et la persécution du roi Siuan la servit. Heou-tsi, de même, n'eut point à souffrir de son délaissement. Lorsque les vers du Che king furent devenus la proie de la critique et que des chroniqueurs, comme Sseu-ma Tsien (101), les eurent transformés en matière historique, on soupçonna que, si Kiang Yuan avait abandonné son enfant, c'était parce qu'elle le méprisait de n'avoir point de père (102), ou bien pour le soustraire à la persécution de l'empereur, son mari, lequel ne savait point d'abord «se conformer aux (ordres du) ciel, ne (les) trouvant point assez clairs »(103). On ne voit rien de tout cela dans le Che king, mais il était naturel que cette explication se présentât, dès que la naissance parut miraculeuse et qu'on ne se rendit plus compte que les pratiques employées par Kiang Yuan pour favoriser sa grossesse étaient d'un usage courant. L'auteur des Annales de Wou et de Yue (104) affirme que Kiang Yuan, quand elle eut marché sur les traces du géant, que son corps eut frémi comme au contact viril et qu'elle se trouva enceinte, eut peur d'être accusée du forfait de débauche. Alors, dit-il ingénieusement, mais à l’inverse du Livre des vers, elle fit des sacrifices pour demander de n'avoir point d'enfant ; comme le ciel ne l'exauça pas, elle exposa Heou-tsi. -Sseu-ma Tsien, plus fidèle à ses documents, quand il met en prose historique la légende de Heou-tsi, se borne à ajouter deux gloses : Parce que c'était néfaste, Kiang Yuan exposa son fils, et (les trois expositions faites) parce que c'était faste, elle le recueillit (105).

Les gloses des auteurs chinois sont intéressantes par l'embar ras qu'elles montrent en voulant expliquer le délaissement du jeune fils du Ciel : elles trahissent un état d'esprit dont aurait pu sortir plus net tement le thème de la persécution du nouveau-né. Leur principal avantage est de faire sentir que, dans ce cas privilégié d'une conception miraculeuse et d'une naissance sans souillure, le nouveau venu a tout de même une épreuve à subir : il lui faut manifester par ses propres moyens, il lui faut faire sa preuve quil mérite 
d'être recueilli et élevé et quỉl n'est point né sans avoir droit à la vie. Dans le grossissement particulier à la légende poétique, l'abandon de l'enfant sur la terre apparait comme une ordalie mythique.

Notons d'abord que l'épreuve est d'un caractère total. D'après les rites, l'enfant, avant d'être porté aux bras, est laissé sur le sol trois jours, et cette période de temps, symboliquement complète, suffit à épuiser la durée : l'enfant en sort complètement éprouvé. Mais que le mythe a de plus puissants moyens d'expression! Heou-tsi, avant qu'il ne soit recueilli, doit être abandonné à trois reprises. Il prend d'abord contact avec la terre habitée, puis avec le sol des forêts, enfin avec l'eau glacée ; abandonné chaque fois dans un milieu qui semble plus hostile, chaque fois son heureuse fortune lui amène des secours plus inespérés. Dans l'étroite ruelle, les boufs et les moutons non seulement évitent de l'écraser, comme Sseu-ma Tsien se borne à dire, mais encore, le Che king l'affirme, ils le protègent et en prennent soin ; perdu dans la forêt déserte, amis inattendus, des bûcherons viennent à lui ; et quand il est laissé sur la glace mince d'un étang — quel secours attendre contre l'e au ou le froid ? - le secours lui vient des airs : un oiseau vole vers lui, qui (c'est le plus touchant dévouement) d'une de ses ailes le soutient et de l'autre le recouvre et le réchauffe. De la triple épreuve, il sort vainqueur.

- Et chaque épreuve lui vaut de nouveaux amis et une alliance plus complète avec la Nature. Sol des villes, terre des bois, eaux des étangs lui sont propices; animaux domestiques, hommes, bêtes sauvages l'assistent. N'est-il pas de la lignée du grand Ancêtre Civilisateur, de la lignée de Houang-ti qui mit la terre en culture et favorisa le développement des oiseaux et des quadrupèdes? Ne faut-il point qu'il devienne Heou-tsi, Prince des Moissons qui a la Vertu d'aider la Nature (106) ? Cette Vertu, il la tient de race, il la manifeste et il l'acquiert. Son génie se forme à l'épreuve : dès quỉl l'a subie, on lui reconnaît, tout enfant encore, une fermeté de montagne, conforme au vouloir d'un géant (car il est né du pas d'un géant) (107). Ainsi l'épreuve avive et met au clair ce qui doit constituer sa personnalité. C'est par une manifestation éclatante de puissance personnelle quil y met fin : quand l'oiseau s'est enfui, Heou-tsi se met à crier de cette voix sonore à laquelle on reconnait une grande âme (108); son intonation de bonne école montre en lui le chef-né : on l'entend loin, on l'entend bien, ses cris emplissent le chemin. Sa mère peut le recueillir ; il fera honneur à sa famille et saura commander en Prince.

Si, dans l'épreuve, au contact de la Terre, sa valeur intime se révèle, sill se voit assister de toutes les espèces d'êtres et fait ainsi alliance avec la Nature, en même temps, par le prodige de cette ordalie splendide, du jugement même du Ciel, il est reconnu son fils. «Le Ciel, qui engendra Heou-tsi, le distingua, par des miracles, des autres hommes, désirant par là rendre manifeste sa Vertu transcendante (109). » Les miracles de cette Nativité, dès qu'on les attribue à la 
volonté du Ciel, sont, de sa part, un aveu de paternité. Lorsque Kiang Yuan relève Heou-tsi de terre, c'est au nom de son père et par son ordre : Heou-tsi est le fils du Ciel. Pour qu'il devînt clair qu’il était bien tel, pour que fût prouvée son essence divine, pour qu'il pût montrer à l'épreuve qu'il avait en lui de quoi devenir le Médiateur des hommes auprès du Souverain d'En -haut, pour qu'il le devînt en effet, il fallait d'abord qu'il restât da ns l'abandon et couché sur la Terre.

L'ensemble de mythes qui constitue la légende de Heou -tsi a une cohésion qui est, pour nous, d'un grand enseignement. Génie agraire, en qui se personnifie la Puissance du Sol cultivé, il tient son pouvoir d'une alliance avec les choses de la Terre, alliance conclue à sa naissance par le fait d'un contact pathétique et prolongé avec les manifestations diverses de la Nature. Héros fondateur d'une race seigneuriale, âme permanente d'un groupe familial à laquelle vont se réunir les âmes individuelles dès que le culte laisse tomber les personnes dans l'oubli, il doit sa pérennité au fait d'avoir été connu par la vertu des rites pratiqués par les communautés autochtones dans leurs Lieux Saints ; associé du Ciel, qui commande aux saisons et pourvoit à la prospérité de l'année, il tient l'emploi de Médiateur auprès de cette Puissance suprême, parce qu'il est le premier d'une Race de chefs chargés par un peuple agricole de promulguer le Calendrier, Loi de l'Alliance des hommes a vec la Nature : ensemble cohérent de croyances où apparaît la marche d'une pensée religieuse dont les créations vont se diversifiant et s'enrichissant - à mesure que de l'idée-souche, fortement et confusément sentie d'une parenté unissant un groupe d'hommes au Sol qui les nourrit, sortent: la conception de Puissances agraires et de Génies civilisateurs, celle d'Ancêtres fondateurs et de Héros éponymes, celle de l'unité du Monde et d'un Pouvoir régulateur ; à mesure qu'à la primitive communauté agricole se superposent la famille fondée sur l'autorité domestique et l'État régi par un Chef. Mais l’idée organique d'une Alliance entre la Terre et l'homme reste au centre de toutes ces croyances : dans le mythe de Heou-tsi, l'exposition sur le sol, par lequel cette alliance se crée, occupe la place d'hon neur; le Héros porte le titre de Prince des Moissons, mais son nom personnel - par lequel sa nature intime est exprimée - est $K^{\prime} i$ : (celui qui fut) Exposé (110).

Vu à travers le développement qu'ont su lui donner la pen sée relieuse et 1a poésie dynastique, le rite du dépôt sur le sol laisse deviner ce qui est sans doute sa raison d'être : c'est une épreuve ; imposée au nouveau venu qui entre dans la vie et pénètre dans un groupe familial, elle crée (tout autant qu'elle suppose) une intime parenté entre le postulant et la Puissance-juge : savoir te Sol nourricier de la Race. 


\section{III}

\begin{abstract}
Il faut encore retrouver et les sentiments sociaux et les structures sociales dont ces faits sont l'expr ession, dont ces mythes ne sont que les représentation, dont ces rites ne sont que les gestes.
\end{abstract}

M. MAUSS, Année sociol., IX, p. 266.

Ordalie mythique ou métaphore d'édification, l'usage d'expo ser l'enfant ne s'est conservé dans la littérature qu'à l'état de concrétion. Peu nombreux sont les textes (c'est là le désavan tage constant des études chinoises), mais, dès qu'on a su en fixer le caractère, ils livrent des faits qui peuvent (ici se trouve le profit de ces études) être mis en leur place dans une série où ils prennent tout leur sens ; - et, de plus, on peut trouver des séries analogues pour y comparer utilement celle qu'on vient d'établir.

Pour y être né, on ne pénètre point d'un seul coup dans le groupe domestique; l'entrée se fait par étapes et, si je puis dire, à mouvements décomposés: le geste initial se prolonge pendant toute la période qu'il inaugure, et la période suivante s'ouvre par un geste nouveau qui la domine. L'initiation à la vie d'un groupe se découpe en une suite de stages, ouverts, chacun, et clôturés par une cérémonie qui, à être à la fois initiale et terminale, prend une apparence ambiguë: elle semble supprimer le passé et créer l'avenir ; les rites qu'on y emploie ont l'air ou bien de tendre à une élimination, ou bien à une création. Stages ou cérémonies, gestes initiaux ou gestes continués, les pratiques s'ordon nent en un mouvement d'ensemble et concourent à une même fin ; elles se conditionnent les unes les autres et se relayent ; réparties dans des temps différents, correspondant à des périodes de durée concrète et de nature singulière, elles forment un tout qui n'est point homogène et qui recèle pourtant une certaine continuité.

Ce n'est qu'à sa $3^{\mathrm{e}}$ année qu'un garçon est mêlé, non point à toute la vie de famille, mais à la vie commune du gynécée : alors il sait parler (111) et l'on met fin par une cérémonie à sa nourriture. - Pendant 3 ans, il a dû vivre dans un certain isolement, confié à la garde de sa mère (ou de sa nourrice) : il a appris d'elle à marcher (un an) et à manger (7 mois). - Au $3^{\mathrm{e}}$ mois (112), l'enfant pris sur les bras par la mère, avait été présenté au père (il savait alors regarder et était capable de rire) : le père, le flattant de la main et le faisant rire, lui donnant, avec un nom personnel, une personnalité et l'intelligence, avait ordonné qu'on prit soin de l'élever : il était alors devenu quelqu'un dont on ne pourrait certes pas porter déjà le deuil, mais à la mort de qui il serait permis de pleurer; ses cheveux avaient été coupés pour la première fois et 
arrangés de manière à montrer symboliquement qu'il deviendrait un fils pieux ; il avait repu de son père la paumée et avait ainsi commencé à lui être affilié; sa mère avait pu le porter sans que pour cela il ait continué d'empêcher les rapports entre son mari et elle : tous deux, après un repas analogue à celui de leur mariage, avaient repris commerce ensemble. La cérémonie avait mis fin à la fois à l'isolement de la mère et à celui de l'enfant.

- Avant le $3^{\mathrm{e}}$ mois, la mère a vécu séparée ; l'enfant, de même, confiné dans une pièce spéciale, seul avec la femme chargée de le porter et de le nourrir. C'est au $3^{\mathrm{e}}$ jour seulement (il avait alors su prouver sa vitalité par ses vagissements) qu'on avait comm encé de le porter ; sur l'ordre du père, averti de la naissance et qui lui-même en avait averti les ancêtres, mais en l'absence des parents, un vassal l'avait relevé de terre et remis à la nourrice : l'enfant avait cessé de jeûner et d'être exposé à terre, quand, dans toutes les directions, on avait dispersé des flèches, avec l'arc exposé, depuis 3 jours, à la grande Porte, l'un des dieux lares de la maison. - Pendant les 3 premiers jours, l'enfant a jeûné, abandonné à terre, tandis que l'arc suspendu signa lait la maison où un garçon venait de naître. - A la naissance, geste dramatique, il avait été déposé sur le sol.

Ce stage de 3 ans qui se décompose en 3 périodes (de la naissance au $3^{\mathrm{e}}$ jour $-\mathrm{du} 3^{\mathrm{e}}$ jour au $3^{\mathrm{e}}$ mois - du $3^{\mathrm{e}}$ mois à la $3^{\mathrm{e}}$ année) dont les premières, les plus courtes, les plus émouvantes, sont ouvertes par le geste rituel le plus puissant, n'est point imposé pour entrer dans le groupe familial au seul nouveau-né. La nouvelle épousée peut toujours être renvoyée dans sa famille (sauf trois cas d'exception), mais il est blâmable de l'abandonner sans raison grave, si le mariage a duré 3 ans $(\underline{113}) ;-$ au $3^{\mathrm{e}}$ mois elle est considérée comme une épouse, au sens plein du mot, et le deuil porté pour elle est complet (114) : car c'est le mois où elle com mence à participer au culte des Ancêtres; on fait alors une cérémonie pour la présenter dans leur Temple (115) ; à la même date les chevaux et les gens de son escorte reçoivent congé et rentrent dans la maison natale $(\underline{116}) ;$ - avant le $3^{\mathrm{e}}$ mois, la nouvelle venue vit dans une espèce de retraite et ne doit point prendre part aux travaux domestiques (117); le $3^{\mathrm{e}}$ jour elle est reçue par les beaux-parents et se sert de l'escalier réservé aux maîtres de la maison (118) : alors cesse, dans sa nouvelle famille, linterdiction de faire de la musique et, chez ses propres parents, on éteint les flambeaux qui brûlaient comme pour un mort $(\underline{119})$; — les 3 premiers jours avaient été, dans les deux familles, des jours lugubres.

Les rites chinois marquent merveilleusement le procédé d'approche ; l'assimilation s'obtient par l'écoulement d'une durée totale (trois jours : 3 est un total), durée qui est dotée d'une effica cité sui generis par un rite initial et qui est emboîtée dans des durées de valeur analogue, mais de nature plus diluée - l'unité de mesure étant accrue, mais le spécificatif nu mérique restant constant - chaque prolongation étant elle-même adaptée à une fin, moins essentielle mais plus complexe, par un rite initial secondaire. De même que les stages de 3 ans et de 3 mois agissent surtout en prolongeant l'effet du stage de 
3 jours et en consolidant les résultats déjà acquis en principe, de même les cérémonies qui les ouvrent servent essentiellement à marquer le chemin parcouru et à donner un élan nouveau à la poursuite de la fin fixée par le rite premier.

De l'espèce d'équivalence fonctionnelle qu'il y a entre les durées emboîtées, il résulte que (sans modifier profondément la valeur des différents stages et étapes) des pratiques peuvent, pour des raisons de convenance, se détacher de l'un des ensembles cérémoniels pour s'adjoindre à un autre. Si les beaux-parents sont morts, la bru ne pourra être reçue par eux le $3^{\mathrm{e}}$ jour, mais, le $3^{\mathrm{e}}$ mois, elle sera présentée à leur Temple. Le fils posthume d'un souverain recevra son nom près de la tablette de son père le $3^{\mathrm{e}}$ mois (comme si le père était vivant), mais, si le mort n'est point enterré, le nom sera donné devant le cercueil, le $3^{\mathrm{e}}$ jour(120). Cette possibilité de déplacement des actes cérémoniels a été certainement utilisée, en bien des cas, pour accorder le schéma rituel avec les changements de conception et d'étiquette qui résultaient d'une nouveauté dans l'orga nisation sociale. Une bonne partie des rites affectés d'abord au $3^{\mathrm{e}}$ jour de la naissance a dû passer au $3^{\mathrm{e}}$ mois, s'agglo mérant alors aux pratiques anciennes de la fête des relevailles, lorsque l'accroissement de la dignité paternelle eut rendu plus redoutable le premier contact entre le père de famille et l'enfant à peine délivré des souillures de la naissance : ainsi les rites veulent que le $3^{\mathrm{e}}$ jour, l'enfant, relevé de terre par le vassal qui le premier le porte, soit reçu de ses mains par sa nourrice, et la mère n'apparaît qu'au $3{ }^{\mathrm{e}}$ mois; mais, puisque les femmes des plébéiens et des nobles ordinaires nourrissaient leurs enfants, elles devaient, sans doute, les recevoir elles-mêmes, au moins dans l'ancien temps, à la cérémonie du début de la nourriture, le $3^{\mathrm{e}}$ jour (121).

L'analyse comparée du procédé d'approche ne met pas seule ment en évidence l'équivalence des stages emboî tés sous l'im pulsion dominatrice du rite initial : elle fait encore sentir que ce rite n'est pas un commencement absolu, mais plutôt un point culminant ou un centre. Au temps de repos des 3 premiers mois, retraite nuptiale, correspond, avant le mariage, une retraite de 3 mois; la fiancée vit alors dans le Temple de sa famille natale. Elle l'abandonne au $3^{\mathrm{e}}$ mois; au $3^{\mathrm{e}}$ mois après les noces, elle peut entrer dans le Temple Ancestral du mari. Les 3 premiers jours du mariage sont lugubres ; 3 jours avant la cérémonie, le fiancé jeûne pour s'y préparer ; sans doute, la future en fait autant. Au bout de cette retraite renforcée, le $3^{\mathrm{e}}$ jour, la fille, pour sortir de la maison natale, prend l'escalier de l'Ouest, celui des étrangers; le $3^{\mathrm{e}}$ jour des noces, elle utilise, dans sa visite aux beaux-parents, l'escalier de l'Est, celui des maîtres de la maison. De même, pendant 3 mois avant l'accouchement, la femme enceinte vit en recluse ; l'accouchée aussi doit rester recluse pendant 3 mois. - Au $3^{\mathrm{e}}$ mois avant la naissance, le mari, s'il est plébéien, abandonne la maison à sa femme ; il y rentre après la cérémonie des relevailles, le $3^{\mathrm{e}}$ mois. Quand la naissance est imminente, il jeûne (le jeûne, en théorie, est de 3 jours) ; pendant les 3 jours qui la suivent, il 
lui faut encore jeûner pour se préparer au sacrifice qui annonce la naissance aux ancêtres. L'enfant passe ses 3 premiers jours à vagir, laissé à terre, sans nourriture; sa mère, pour sa nourriture, pour son coucher, pour la musique qu'elle entendait, s'était, dans son intérêt, soumise, les 3 der niers mois de la grossesse, à de nombreuses interdictions (122).

L'acquisition de qualités nouvelles, qui méritent linncorpora tion à un groupe défini, ne peut se faire sans que d'abord soient mises au clair les qualités anciennes que les nouvelles vont recouvrir au point de sembler les supprimer. Avant de quitter sa famille, l'épousée doit se pénétrer de l'influence qui émane du lieu le plus sacré de la maison natale: le droit qu'elle a d'entrer par mariage dans une famille se voit quand elle a rendu manifeste le fait qu'elle appartient à une famille d'un autre nom. Le rite central de l'entrée dans un groupe est précédé de pratiques qui semblent, à première vue, préparer cette entrée en rompant les attaches avec un autre groupe, mais ces attaches ne sont jamais toutes rompues : linfluence de la cérémonie centrale, même propagée pendant une triple durée totale à travers les stages successifs et les rites d'étape, n'efface point les qualités anciennes qui demeurent une condition à l'acquisition des nou velles; la femme mariée est désignée, jusquà la mort, par le nom de sa famille originelle ; même éteints les flambeaux qui, dans la maison natale, semblent l'emblème d'une mort, même renvoyée l'escorte qui l'a conduite, elle reste la fille de tels parents, elle leur doit des visites, elle leur doit le deuil (123) ; si elle est répudiée (124), elle ne deviendra pas sui juris, mais retombera sous leur autorité (125) - elle ne pourrait plus être répudiée, si sa famille natale était détruite. Le procédé d'approche semble se doubler d'un procédé d'éviction et l'entrée supposer une sortie: en fait, l'accroissement de personnalité, l'acquisition d'un sacré nouveau, s'accompagne bien de pratiques par lesquelles un autre sacré s'élimine - tout momentanément et seulement pour prévenir un mélange - mais par lesquelles aussi ce fonds ancien s'affirme et, d'une manière sous-jacente, se consolide.

L'entrée d'un nouveau -venu dans un groupe y détermine un trouble et une émotion qui vont se propageant comme des ondulations concentriques toujours plus faiblement marquées; la première vague d'émotion qui se li mite au cercle le plus étroit et à la plus courte durée, est aussi la plus franchement dessinée ; l'émotion centrale propagée dans un temps plus long et dans un milieu plus complexe prend à mesure un aspect moins simple et des traits moins saillants, mais c'est toujours la même émotion ; si les périodes successives de sa manifestation apparaissent comme autant d'ensembles hétérogènes, elles ont entre elles une espèce de parenté rythmique qui se décèle au coefficient numérique de leur durée. Le progrès correspondant à la première période et au geste initial qui, continué tant qu'elle dure, la constitue, ne peut pas être entièrement différent des progrès postérieurs quill conditionne : sous les dépassements réalisés par eux et par lesquels le progrès précédent semble être oblitéré, il doit être possible, si l'on a saisi le rythme de 
cette progression (qui se manifeste par vagues successives et qui, pourtant, est continue), de retrouver l'efficacité particulière de ce premier moteur qui donne sa loi au mouvement d'ensemble.

Or, remontons, en le prenant assez loin, le cours de la vie d'un garçon. A 30 ans, il se marie, reçoit un emploi à la cour seigneuriale et possède une maison hors de l'habitation paternelle (126) ; il demeure à part, car son mariage et son inféodation lui assignent dans la société une place distincte ; la cérémonie de majorité qui a rendu possibles et l'inféodation et le mariage, est celle même qui, à 20 ans, l'a affilié à son père (127) : dès qu'ont été définitivement acquises les qualités qu'impliquent les rap ports de fils à père, s'est ouverte la possibilité d'acquisitions nouvelles ; le fils gagne le moyen, non pas, certes, de sortir du champ dinfluence de la parenté agnatique, mais, du moins, de pouvoir pénétrer dans un milieu où jouent des influences plus complexes; il échappe en partie à l'exclusive puissance paternelle par les conséquences mêmes de la cérémonie qui a fondé cette puissance. - Avant cette cérémonie qui a fait de lui un homme et un fils, avant 20 ans, il n'a point du tout vécu dans le cercle d'action de la famille agnatique et de la puissance paternelle (celle-ci n'a révélé son empire futur que par des signes prémonitoires) et il a dû porter à la ceinture le sachet de parfums distinctif de la toilette féminine (128) (sa virilité ne s'est jusqu'alors manifestée qu'en quelques occasions notables). Mais à la cérémonie de la 20e année, quand le père, faisant de lui un membre majeur du groupe familial, lui a fait donner, avec un habit d'homme fait, un nom viril, le rite achevé, il est allé rendre visite et faire offrande, sur la porte du gynécée, à sa mère qui l'a salué la première (129). Or, depuis ses 15 ans (puberté), un garçon est à l'âge où il peut, dans les cérémonies du culte féodal, prendre la place que tient une fille dans les fêtes populaires (130) : c'est donc après que s'est révélé en lui, avec une force telle qu'on a pu l'utiliser ritu ellement, un certain fonds de nature féminine, quill abandonne enfin ses attributs féminins. De 10 à 20 ans, le garçon a fait son éducation hors de la maison paternelle, non point sous la direction du père ou de ses parents en ligne masculine, mais confié soit à des anciens, soit à la famille de sa mère (131) : il est reçu dans la classe des jeunes gens, il est accepté dans la famille agnatique, seulement quand ont été mis au clair les liens qui l'unissent aux parents maternels et affirmée l'espèce de parenté qu'il y a entre vieillards et enfants (une même alimentation leur convient (132) ; garçons et filles peuvent s'asseoir sur la même natte à moins de 7 ans (133); à plus de 70, un mari peut serrer ses effets personnels aux mêmes endroits que sa femme) (134). - Si le garçon est envoyé à 10 ans hors de la maison paternelle, jusque-là il doit vivre dans la partie la plus reculée de la demeure; - jusqu'à 7 ans, il reste dans le gynécée, complètement mêlé aux filles. - Pendant les trois premières années, il n'a de contact qu'avec la mère qui l'élève $(\underline{135})$ (au $3^{\mathrm{e}}$ mois le père lui donne la paumée et on le coiffe en garçon). - Pendant les 3 premiers mois, il n'a de contact qu'avec la femme qui le nourrit (au $3^{\mathrm{e}}$ jour, un vassal du père le porte, et, peur lui, l'on tire de l'arc, arme virile). - Pendant les 3 premiers 
jours, il n'a de contact qu'avec la Terre (on l'expose un moment sur 1 e lit du père dé famille) (136).

- Sous les Tcheou ( $3^{\mathrm{e}}$ dynastie royale), on enterrait les enfants morts de 16 à 19 ans dans le cercueil qui était d'usage commun sous les Yin $\left(2^{\mathrm{e}}\right.$ dynastie); ceux qui mouraient de 8 à 15 ans dans le cercueil employé sous les Hia (ire dynastie); ceux enfin qui mouraient de 3 mois à 7 ans dans le cercueil en usage dans les temps antérieurs aux 3 dynasties royales (137) : aux plus jeunes convenaient les rites les plus anciens. De même, en avançant en âge, un jeune Chinois, comme s'il refaisait pour son compte les étapes de la civilisation de son pays, n'arrivait à être incorporé dans la famille agnatique et le groupe féodal qu'après avoir subi l'influ ence conservée de l'ancienne famille utérine et de l'ancien mode de groupement où l'autorité appartenait aux anciens; il ne se rapprochait du père et ne vivait au milieu des hommes qu'après avoir passé, sous l'autorité de sa mère, ses premiers jours parmi les femmes; et, avant tout autre apparentement, c'était à la Terre quỉl avait d'abord fallu le présenter.

De la même manière que les révolutions sociales, d'où étaient sorties les structures nouvelles, n'avaient point oblitéré complètement les formes anciennes qui avaient conditionné leur avènement, de la même manière les sacralisations récentes (qui dans la vie enfantine correspondent à ces révolutions), tout en impliquant des désacralisations qui semblent éliminer les qualités d'abord acquises au mo ment où elles apparaissent portées à leur point de perfection, de la même manière ces sacralisations, qui marquent les divers sommets de l'onde de sacrement déterminée par le rite central de la naissance, ne font, essentiellement, que manifester la puissance propagée de ce rite, puissance affirmée par les créations complexes quill conditionne. Déposer l'enfant à terre et l'y laisser trois jours, c'est préparer sa présentation à sa nourrice, à sa mère, aux femmes de la maison, au père, aux parents paternels, aux membres du même groupe féodal ; - c'est le mettre à même d'acquérir la qualité substantielle qui lui per mettra d'entrer dans différents systèmes de relations; - c'est créer en lui une aptitude à des apparentements divers ; - c'est le présenter à une Puissance qui est l'origine de tous les liens humains et le premier principe de la cohésion sociale : la Terre natale.

Ainsi, la Terre est créatrice de parenté et d'affiliation. Pour quoi ? Et pourquoi, d'autre part, est-elle conçue comme une nourrice, comme une puissance féminine qui confère des attributs féminins ? Et, puisqu'il ne faut voir dans aucun rite un commencement absolu, puisque tous impliquent une progression et comme une sortie hors d'un état antérieur, de quoi sépare -t-on l'enfant quand on le dépose sur le sol ?

A mesure que l'enfant pénètre dans la famille, sa personnalité, incomplète d'abord, se constitue : le houen, lâme-souffle, ne lui vient qu'après le p'o, lâme corporelle ; les premiers jours il ne sait que pleurer; à 3 mois il peut rire 
et il sait voir ; à 7 mois, les dents poussent, il peut manger ; à 1 an, il marche ; à 3 ans, il parle ; à 7 ans, il change de dents ; à 15 ans $(-(7 \times 2)+1)$ il est pubère (138). L'embryon, 3 mois après la conception, est formé ; ayant atteint sa pleine maturité, à 7 mois, il se prépare à venir au monde (139).

A 70 ans, le vieillard se prépare à quitter le monde (140). Quand il meurt, il abandonne, par étapes, sa famille et sa personnalité. Le mourant, comme l'enfant naissant, est déposé sur le sol. Lorsque l'on a recueilli le dernier souffle sur de l'ouate, quand on a en vain rappelé l'âme -souffle qui, la première, s'en va, tous pleurent autour du mort couché sur la terre (de même, sur le sol, pendant 3 jours, l'enfant vagit) ; comme on met le nouveau-né sur le lit, on y met le mort et on l'y habille ; au mort, comme au petit garçon, on donne un talisman de jade : on le lui fait tenir dans la bouche. La maison du mort est signalée comme celle de la naissance, non point, cependant, par un simple attribut sexuel, mais par une bannière où sont inscrits le nom de famille et le nom personnel qui définissent le défunt (141). - Au 3 jour, on le porte au cercueil et, non pas dans la chambre, mais près des degrés par où s'en vont les hôtes; on l'enterre, légèrement, et la tête vers le Sud comme un vivant (142). - Au $3^{\mathrm{e}}$ mois, on le transporte, pour l'y enterrer profondément, la tête au Nord, au Nord de la ville, au cimetière de famille, où il retrouve tous les autres Ancêtres. - Trois ans la maison reste vide : le fils ne peut venir l'habiter qu'à la fin du deuil. Ainsi, après des rites parallèles à ceux de la naissance et qui durent 3 jours, il se fait, au 30 jour et au $3^{\mathrm{e}}$ mois, deux cérémonies d'enterrement, comme il y a, pour l'enfant, au $3^{\mathrm{e}}$ jour et au $3^{\mathrm{e}}$ mois, deux cérémonies de réception. Si le nouveau-né n'est vraiment chez lui dans la maison qu'au bout de 3 ans, c'est aussi a u bout de 3 ans que le mort n'y est plus le maître. Sous l'impulsion du rite initial commun, par les effets développés du dépôt sur le sol, l'enfant entre dans la famille et le défunt en sort (143).

C'est mal dire : le défunt ne sort pas de la famille ; il ne fait que délaisser la communauté des vivants; du même pas qu'il s'en éloigne, il approche de la communauté des Ancêtres. Sitôt le souffle recueilli, sitôt le houen rappelé, on fait une tablette provisoire, valable tant que dure l'enterrement provisoire, et qui, comme le corps du mort, est gardée dans la maison. Un peu après l'enterrement définitif, lorsque le corps est réuni à celui des aïeux, on fait une tablette définitive que l'on place dans le Temple Ancestral ; mais jusqu'à la $3^{\mathrm{e}}$ année, elle n’y a pas de chez soi : elle est associée à celle du grand-père. Ce stage écoulé, le deuil fini — quand le mort n'est plus le maître de la maison -, il reçoit, avec sa tablette, une place à lui dans le Temple Ancestral ; il y jouit d'honneurs particuliers: il a acquis, dans le monde des Ancêtres, une personnalité (144). - Celle-là aussi doit périr 3 ans après la mort de son descendant, à la cinquième génération (souche comprise), la tablette de l'Ancêtre perd ra sa place et ses honneurs particuliers ; réunie aux tablettes des plus anciens aïeux, elle sera conservée auprès de celle du fondateur de la famille $(\underline{145})$ : dès lors, le défunt, corps et âme, a fait retour à la masse 
ancestrale, et son nom, dont l'emploi cesse d'être interdit dans la parenté (146), peut servir à donner rang et personnalité, dans la famille vivante, lors de la cérémonie du $3^{\mathrm{e}}$ mois, à un nouveau-né (147).

La tablette est le centre du culte ancestral ; mais, pour rendre plus sensible, dans les cérémonies, la présence de l'Ancêtre, on devait lui constituer un représentant vivant: ce ne pouvait être qu'un de ses descendants; encore fallait-il qu'il appartînt à la même moitié de la famille, comme c'est le cas, par exemple, pour un petit-fils (148), - les membres d'une famille étant répartis en deux groupes par générations alternées. Le chef du culte, pour rendre hommage à son père, s’inclin ait devant son propre fils et le servait comme il eût fait pour son père (149) (fait extraordinaire, selon les règles de la morale classique !) : c'est qu'il voyait alors en son fils le représentant de son propre père. Et l'on dev ait porter le deuil du fils pour le père à la mort d'un fils aîné, « corps véritable de l'ancêtre » (150).

- Ces usages supposent une croyance à la réincarnation des morts dans leur propre famille. Les textes en donnent quelques témoignages directs : Pao Sseu est une réincarnation des princes de Pao (151); • la naissance du roi Mou est le fait d'un prince de Fang, aïeul de sa mère (152); celle du comte Lan (orchidée) de Tcheng, le fait d'un prince de Yen, ancêtre, lui aussi, de sa mère (153) : dans ce dernier cas, ce n'est point seulement la vie qui est donnée par l'ancêtre, mais aussi, emblème végétal, le nom personnel.

La naissance de Lan (orchidée), seigneur de Tcheng, nous est contée d'une manière qui rappelle avec précision les pratiques des fêtes du mariage dans le pays de Tcheng. Lorsque les auteurs chinois voulurent expliquer pourquoi, dans ce pays, les couples de fiancés cueillaient des orchidées sur les bords de la Wei, ils dirent qu'en passant la rivière avec ces fleurs on se proposait de rappeler, par le geste et la voix, l'âme-souffle, le houen, pour l'unir au p'o, à l'âme corporelle. Les penseurs, en effet, avaient admis, dès leurs plus anciennes spéculations, que la personnalité humaine résulte de l'union d'un houen à un p'o, que la rupture de cette union, le houen partant le premier, opère la mort, et que son établissement, le houen arrivant le dernier, donne la vie (154).

Le mourant, étant placé à terre, lorsqu'on avait recueilli son dernier souffle, tous pleuraient autour de lui ; puis quelqu'un montait sur le toit, et, tourné vers le Nord, rappelait le houen, pour qu'il revint s'unir au p'o, en agitant les vêtements du défunt et en criant, par 3 fois, son nom personnel $(\underline{155})$. A la cérémonie du $3^{\mathrm{e}}$ mois, où le cercueil est définitivement et profondément mis en terre, un sage, vanté pour son sens du cérémonial (156), fit 3 fois le tour de la tombe en poussant des cris et en disant :

«Que la chair et les os retournent à nouveau à la Terre ! c'est le Destin ! mais le souffle-houen, il n'y a pas d'endroit où il ne puisse s'en aller ! il n'y a pas d'endroit où il ne puisse s'en aller ! 
Et lui-même, il s'en alla : il avait, par ce rite, profondément scellé et triplement enclos dans la terre ce qui devait lui revenir ; et, définitivement, il en avait disjoint le houen, l'âme-souffle, qu'il espérait bien, laissant le corps avec celui des ancêtres, ramener avec lui pour lui faire habiter le Temple Ancestral, supportée par la tablette définitive. L'ouvre, alors achevée, n'avait-elle point été inaugurée et réalisée en principe par les rites des premiers jours ? Le dépôt sur le sol, la criée, le rappel du houen, obtiennent cette dissolution de la personnalité que la pensée consciente se représente comme une séparation de l'âme-souffle et de l'âme corporelle, et comme un retour, retour à la terre commune des parents, retour à la demeure commune des aïeux. Sans doute le retour se fait-il d'une marche moins simple et moins rapide pour les parties hautes de la personnalité (dont le souvenir, plus vivace, est conservé, pendant quatre générations, par le culte des ancêtres quand celui-ci s'est établi) ; mais, enfin, l'essentiel de la mort, que réalisent les premiers rites, est d'être un procès de désincarnation et de retour à la masse ancestrale.

Inversement, la naissance, résultat d'une incarnation d'an cêtre, implique un procédé d'éviction et le délaissement de la masse ancestrale. Naître, c'est, d'abord, sortir de la commu nauté des morts : le naissant meurt à la famille des Ancêtres comme l'épousée meurt à sa famille originelle ; d'où le caractère lugubre des premiers jours et des jours symétriques qui les précèdent; d'où cet abandon du nouveau-né et ses pleurs.

Naître, c'est encore passer d'une existence indistincte à une vie individualisée ; c'est acquérir une personnalité. Pour empê cher que celle du mourant ne se dissolve et, aussi bien, pour constater qu'elle s'est dissoute et pour en prendre acte (157), les parents hurlent à la mort et crient le nom personnel, rappelant ainsi le houen pour l'unir au p'o. La personnalité est d'autant plus lente à se former que l'organisation sociale est plus complexe dans la famille agnatique, l'enfant n'en a vraiment g agné une (et encore point complètement) (158) qu'au $3^{\mathrm{e}}$ mois, quand il reçoit de son père (qui l'excite à rire) un nom personnel. Mais au temps qu'évoquent les mythes, c'est Kiang Yuan, c'est la mère, qui relève Heou-tsi de terre et qui, à ce moment même, lui donne son nom personnel (159). Heou-tsi met fin à son épreuve par ses vagissements sonores; les garçons de race royale que l'on expose sur le lit pleurent bruyamment, et l'on s'en réjouit comme d'un heureux présage; quand le grand $\mathrm{Yu}$ veut rappeler d'un trait la naissance de son fils (160), il se borne à reproduire l'onomatopée qui peint les premiers cris. Ces cris de l'enfant exposé les 3 premiers jours (comme les rires du $3^{\mathrm{e}}$ mois) marquent, en effet, l'apparition d'une personnalité qui mérite d'être définie par un nom : l'âme, c'est le nom et c'est la voix (161). Quand il hurlait à la naissance, le nouveau-né prouvait sa vitalité, affirmait son droit à la vie, ou, si l'on veut, rendait manifeste à la fois et confirmait la réunion au p'o de ce houen qui vient en dernier lieu et qu'on pense pouvoir attacher au $p^{\prime} o$ en criant le nom personnel. Alors la réincarnation était parfaite et paraissait valable ; l'épreuve 
était subie et la preuve donnée du retour à la vie, de la rentrée dans la famille des vivants.

Qu'il y ait formation ou dispersion d'une personnalité, procès d'incarnation ou de désincarnation — quel que soit le sens du changement le fait essentiel est toujours le passage d'une section de la parenté dans une autre. Pour naître ou pour mourir, pour entrer dans la famille vivante ou dans la famille ancestrale (et pour sortir de l'une ou de l'autre), il y a un seuil commun, la Terre natale. Elle n'est point seulement le lieu où s'inaugurent la vie et la survie, elle est aussi le grand témoin de l'initiation au genre d'existence nouveau : elle est le pouvoir souverain qui décide le succès de l'ordalie latente dans cette initiation. La Terre natale apparaît comme une Puissance d'où émanent les Vertus caractéristiques d'une Race, qualités communes à la masse confuse des aïeux et au groupe complexe des vivants, et dont il faut d'abord qu'on se pénètre si l'on veut passer dans l'un ou l'autre groupement. Quand on dépose sûr la Terre le nouveau-né ou le mourant, c'est à Elle de dire si la naissance ou la mort sont valables, s'il faut les prendre pour des faits acquis et réguliers. C'est à la Terre que l'on demande de définir le statut de chacun des membres de la famille : elle est le juge suprême qui assigne à chacun la place qui convient. Si personne ne se hasarde à porter l'enfant avant que la Terre ne l'ait porté, si, pour avoir le moyen d'acquérir plus tard les qualifications multiples que supposent les relations humaines, celui-ci (comme le mourant) doit d'abord s'imprégner, au contact du Sol natal, de ses qualités essentielles, si ce rapprochement avec la Terre apparaît comme la condition première de tout apparentement, qu'est-ce à dire sinon que la Terre natale est la grande Parente ? - et que le fond de toute solidarité est la solidarité territoriale ? Déposer sur le Sol, pour que ce maître souverain du rite de la naissance et de la mort, à qui l'on fait appel, fasse le départ de ceux qui doivent ressortir de la masse ancestrale ou de la famille vivante et les sépare soit de l'un, soit de l'autre groupe, c'est (affirmant que le principe premier de toute cohésion est une consubstantialité territoriale) vouloir, par ce contact solennel aux moments critiques du cycle de morts et de renaissances qui constitue toute existence, recréer, pour chaque individu, cette consubstantialité essentielle.

- Le rite du dépôt sur la Terre implique l'idée d'une identité su bstantielle entre la Race et le Sol. Cette idée se traduit, en effet, par le sentiment d'autochtonie qui est le plus vif de ceux que nous pouvons saisir aux débuts de l'histoire chinoise (162) ; l'idée d'une alliance étroite entre un pays et ses habitants est une croyance si profonde qu'elle est restée au cour des institutions religieuses et du droit public (163). Elle s'exprimait anciennement par le plus vieux des cultes de la Chine, celui des Lieux Saints. Mais cette alliance implique des rapports d'un ordre plus général que ne sont les rapports de famille; si la consubstantialité territoriale est au fond de la consubstantialité domestique, elle la dépasse : et, en effet, portée par l'onde 
rituelle que propage le geste du dépôt sur le sol, l'enfant pénètre dans le groupe des parents, puis aussi dans le groupement de familles qu'est la société politique. Les fêtes des Lieux Saints n'étaient point les fêtes particulières d'une famille, mais celles de toute une communauté agricole ; la solidarité qui s'établissait à leur occasion correspond à une organisation essentiellement territoriale où ne peut déjà avoir grande force l'idée de parenté, du moins en tant qu'elle implique celle de filiation et de lignée a ncestrale. Mais quand le culte des Lieux Saints, principes d'alliance et réservoirs collectifs des âmes d'un Pays, prend la forme d'un culte des Centres Ancestraux (164), demeures des âmes d'une famille -, quand la famille organisée est fortement attachée au sol qu'elle cultive - la représentation que l'on se fait des liens unissant l'homme à la Terre s'enrichit de valeurs nouvelles ; le sentiment, confus et profond, d'une consubstantialité s'accompagne alors de celui d'une puissan ce génératrice.

Peut-être peut-on comprendre à quoi tient ce développement de l'idée-souche. Les enfants étaient enterrés selon de plus vieux usages, on l'a vu, lorsquils mouraient plus jeunes (165). Aux temps de la troisième dynastie, les enfants morts entre 8 et 11 ans étaient enterrés, selon les rites de la première dynastie (Hia), dans le jardin (166). Il y a de grandes chances que l'enterrement dans l'enclos domestique, ou même dans la maison, ait ét é la coutume ancienne. D'après les rites des Yin (deuxième dynastie), on devait faire participer à la mort les cinq dieux lares de la maison et cela par des pratiques qui impliquent une espèce de destruction symbolique (167). La demeure, en tout cas, devenait inhabitable pendant le deuil ; les survivants devaient l'abandonner et se construire une cabane (168). Aux temps historiques, si l'enterrement définitif se fait hors de la maison, l'enterrement provisoire continue de s'y faire et c'est l'essentiel : le mort se désincarne inhumé dans le sol domestique (169); le corps retourne à la Terre ; l'âme (au moins celle de ceux qui ne sont point morts avant l'âge viril, et qui ont eu des descendants, de ceux qui avaient la puissance d'engendrer ou la capacité paternelle) hante le coin sombre de la maison où est le lit du père de famille (170). Quoi d'étonnant si, une fois établies les croyances qui sont à la base du culte des Ancêtres, le Sol domestique, principe reconnu de consubstantialité dorénavant conçu comme le séjour des âmes ancestrales attendant leur réincarnation, soit apparu comme doué d'un pouvoir générateur (171)?

Mais le coin sombre que hantent les âmes des auteurs de la race est aussi le grenier où l'on conserve les semences (« enclose en elles est de la vie » (172), disent les hymnes) qui lèveront dans le champ domestique. Quoi d'étonnant si les idées mêlées de puissance procréatrice et de puissance nourricière sont devenues les attributs de la Terre natale?

- La Terre natale, parente, féconde, nourricière a, en outre, des attributs féminins ; c'est une mère, c'est la Mère. Au temps des Tcheou, les graines destinées -à ensemencer le champ royal n'étaient point conservées dans la 
chambre du Fils du Ciel, mais dans les appartements de la Reine : elles l'étaient à titre de présage d'une belle descendance, et la Reine avait pour charge, aidée des autres femmes royales, de faire germer ces grains (173). Si, dans la maison noble, le père de famille met son lit où sont les semences et où hantent les âmes, c'est qu'il a usurpé la place de la mère de famille. Il fut un temps où la famille était utérine et où un mari, dans la maison conjugale, n'était qu'un gendre (174); l'usage s'en est longtemps conservé. Quand on ne le pratiquait plus, il restait encore que l'habitation était chose féminine, dont l'homme n'occupait vraiment que les abords extérieurs (175). Chez les gens du peuple, quand mari et femme, aux environs d'une naissance, doivent rompre pour un temps leur union, c'est le mari qui vide la place, c'est la mère de famille qui l'occupe (176). Et, d'ailleurs, presque tous les cas de réincarnation que nous connaissons ne sont-ils point le fait d'ancêtres de la femme (177) ? Ainsi, parce que, lorsque la famille reçut une forte organisation, la filiation reconnue était la filiation utérine, la Terre, quand ils l'imaginèrent douée d'un pouvoir générateur, devint pour les Chinois la Terre-mère.

- Il y a, dans les représentations que les Chinois se sont faites de la Terre, une diversité qui s'explique parfaitement. Elle apparut, d'abord, sous l'aspect neutre du Lieu Saint, comme le principe de toute solidarité ; représentation qui correspond à un sentiment intense et vague d'autochtonie et à une organisation essentiellement territoriale. Dans l'ensemble de conceptions et d’images que détermina une organisation de la famille fondée sur la filiation utérine, la Terre domestique fut aperçue sous les traits d'une puissance maternelle et nourricière. Avec l'apparition de la famille agnatique et du pouvoir seigneurial, le Sol revêtit l'apparence virile des chefs qui se le partageaient, génie du fief, génie du lit du maître de maison. Mais, à cet âge encore, le sentiment subsista de la Terre-mère et, quand le culte impérial du Ciel eut fourni un soutien sublime à l'idée de puissance paternelle, la Terre retrouva par opposition, de façon éclatante, tous ses attributs féminins (178).

La même évolution explique les modifications survenues dans le rituel de l'enfance et dans la pratique du dépôt sur le sol. A l'époque classique, c'est par l'ordre du père, chef de culte, que s'opèrent les cérémonies diverses de la réception dans la famille ; c'est lui qui donne le nom personnel ; l'affiliation de l'enfant au père, fait récent, étant lente à obtenir, la dation du nom est reculée au $3^{\mathrm{e}}$ mois ; le $3^{\mathrm{e}}$ jour, le père n'est représenté que par un vassal à la cérémonie du portage; la mère elle-même n'y est point présente, écartée qu'elle est de son enfant par une espèce de participation à la dignité maritale ; dans les hautes classes de la noblesse, elle ne peut plus le nourrir ; pour elle, comme pour le père, les intermédiaires se multiplient (179). Des rites d'approche sont devenus nécessaires pour créer l'affi liation agnatique : au dépôt sur le Sol s'ajoute, pour le garçon, l'exposition sur le Lit, génie du lieu, masculin, qui le virilise et en fait un fils de famille; dans la joie des naissances mâles, cette exposition semble l'essentiel du rite et prend un air de 
parade. Mais, grâce aux filles qui ne pénètrent point, pour ainsi dire, dans le cercle agnatique et qui doivent quitter la terre domestique, se maintient le souvenir du rite ancien : l'abandon pendant trois jours sur la Terre-mère, féconde et nourricière. 


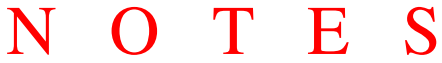

(1) Mme DE MAINTENON (Entretiens sur l'éducation des filles, du mois de mars 1703 ; glose sur le Proverbe, XXXI, 19: Elle met ses mains au fuseau et ses mains tiennent la quenouille).

(2) Nom sous lequel est connue Pan Tchao, sœur et auxiliaire de l'historien Pan Kou ; Ts'ao est le nom de famille de son mari, $\mathrm{Ta}$-kou le titre des fonctions dont elle fut chargée dans le gynécée impérial. On trouvera au chap. CXLV du Heou han chou sa biographie ainsi que le texte des Avertissements aux femmes.

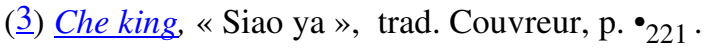

(4) La Tsa'o Ta-kou a glosé sur le texte en utilisant les traditions que l'on retrouve dans les notes des commentateurs du Che king. Tcheng K'ang-Tch'eng indique, par exemple, que le garçon est placé sur le lit pour manifester sa dignité, la fille à terre pour marquer son humilité. L'expression qui signifie «(objet de) terre cuite» dans le texte de Pan tchao est une expression double composée du mot employé par le Che king et du mot par lequel Tcheng l'inter prète. Tcheng ajoute qu'il s'agit là d'un instrument servant aux tisserandes (fuserole ?). - Habit de jour : $\mathrm{m}$. à $\mathrm{m}$. vêtement inférieur, emblème de la règle qui veut qu'un homme ne s'occupe que des affaires de l'extérieur de la maison (Tcheng). - Sceptre en jade : tablette d’investiture tenue en main aux céré monies des cours seigneuriales (Tcheng, Cf. Tcheou li, « Ta Tsong-po », Biot, I, p. 431 et 432). - Genouillères rouges : signe distinctif du roi et des seigneurs (Tcheng, Cf. Li ki, Couvreur, I, p. ${ }^{701}$ ). - Maison royale ou fief princier: les fils du roi Siuan auront un apanage sauf l'aîné qui succédera (Tcheng). — Habit de nuit: emblème de la règle qui veut qu'une femme ne s'occupe que des affaires de la maison intérieure (Tcheng). Selon Mao, langes. - Rien pour le mal, une femme ne fait rien de sa propre autorité. - K' ong Ying-ta indique que le garçon est placé sur la terre à sa naissance, tout comme la fille : quand on le met xur le lit, ce n'est plus le tout premier moment de la naissance.

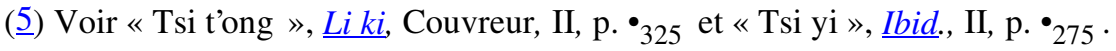

(6) Voir Che king, «Chao nan », 2 et 3, Couvreur, p. • 18 et ${ }_{19}$, Cf. « Tsi t'ong », $\underline{\text { Li ki}}$, Couvreur, II, p. $\bullet_{321}$.

(7) Thème général des préfaces du Tcheou nan et du Chao nan (premières sections du Kouo fong dans le Che king. Voir Granet, Fêtes et chansons anciennes, Cf. encore Ta ya, I, 6, Che king, Couvreur, p. $\cdot 333$.

() Formule de la répudiation, « Tsa ki », Li ki, Couvreur, II, p. • 197 sqq

(9) Voir par exemple, SMT, IV, 52.

(10) Voir Granet, Polygynie sororale, Conclusion.

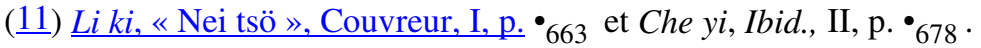

(12) Voir Granet, Coutumes matrimoniales, et Chansons anciennes.

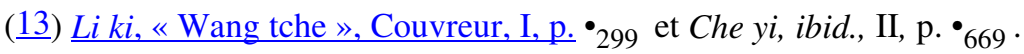

(14) Les caractères 'bru' et 'épouse' notent graphiquement l'impor tance de la serviette pour

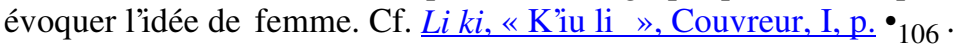


(15) Cf. «Yue ling », Li ki, Couvreur, I, p. $\bullet_{350}$ et Tsi t’ong, ibid., II, p. • 322 •

(16) «Nei tsö », Li ki, Couvreur, I, p. ${ }^{6} 663$.

(17) $\underline{\text { Ibid., pp. }} \cdot{ }^{\bullet} 663-664$ et ${ }^{672}$ (Voir au Tsouo tchouan, Houan, $6^{\text {e }}$ an., Legge, p. 49, un exemple historique des cérémonies de la naissance).

(18) $\underline{\text { Ibid., p. }} \cdot 665$.

(19) Glose de Tcheng.

$(\underline{20}) \lll$ Nei tsö », Ibid., pp. $\bullet 665$ sqq $\bullet$

(21) $\underline{\text { Ibid, p. }}{ }_{672}$. La fin de la nourriture donne lieu à une petite cérémonie ; la nourrice du fils d'un seigneur, quand on la renvoie, est reçue par le seigneur et gratifiée d'un présent.

(22) Ibid., p. ${ }_{663}$.

(23) On va voir quiil est l'arbre consacré au Centre et correspond à l'élément Terre. Voir encore Chansons anciennes, les références données.

(24) Cf. Chansons anciennes, XLVI, v. 20 et note aaa, note bbb. La pièce où se retire la femme avant d'accoucher est dite « chambre de repos» («Ta tai », Li ki, chap. XLVIII) et « chambre de l'armoise » (Sin chou,chap X),

(25) K' ong Ying-ta (glose au « $\underline{\text { Che yi } », L i ~ k i, \text { Couvreur, II, p. }}{ }^{\bullet} 678$ ).

(26) Note de Tcheng au « Nei tsö », Li ki, Couvreur, I, p. ${ }_{663}$.

(27) Par exemple : Fong sou t'ong yi, un arc de pêcher et des flèches d'épine servent à chasser les Calamités (au même passage, l'on voit que, quand le sage Yi Yin entra au service de T'ang le victorieux, fondateur de la dynastie Yin, celui-ci prit soin de le faire fumer sur un feu d'armoise : l'armoise de 1a chambre de l'accouchée correspond -elle à des fumigations et à un feu de l'accouchée ?) De même Kou kin tchou chap. I, mêmes procédés pour chasser le Néfaste. Comp. Tchong houe kou kin tchou.

(28) Voir Granet, Coutumes matrimoniales, la glose de Tcheng.

(29) Chouo wen. Une impureté résulte des écoulements sanguins, menstrues, avortements, accouchements.

(으) Voir Tsouo tchouan. Tchao, 12e an. Legge, p. 641 et Chavannes, SMT, IV, 361.

(31) « Che yi », Li ki, Couvreur, II, p. ${ }_{678}$ et glose de Tcheng au « Nei tsö », Ibid., I, p. 663 [css cf. note 26]

(32) « Che yi », ibid.

(33) Inséré dans le Sin chou, l.c. L'arc correspondant à l'Est est en eløococca [= (saison) Printemps $=($ animal d'offrande $)$ Coq $] ;$ l'arc du Sud en saule $[=$ Eté $=$ Chien $] ;$ l'arc du Centre en mûrier [= Bouf $]$; l'arc de l'Ouest en jujubier [= Automne = Mouton $]$; l'arc du Nord en plaqueminier [= Hiver= Porc].

(느) «Nei tsö », Li ki, Couvreur, I, p. $\cdot{ }_{671}$.

(35) Coin situé sous la fenêtre du Sud, ne recevant pas directement la lumière et peu éclairé.

(흐) Le mot ngao a le sens de grenier.

(그) « Tseng tseu wen », $L i k i$, Couvreur, I, p. ${ }_{452}$ (l'angle SW s'oppose à l'angle NW qui reçoit toute la lumière de la fenêtre). 
$(\underline{38})$ « K’iu li », Li ki, Couvreur, I, p. • ${ }_{13}$, Cf. Ibid., II, pp.• 388-389. Le coin SW et les degrés de l'Est sont les places rituelles du maitre de maison.

(9) On se mariait hors de la famille et dans le pays (Cf. Fêtes et chansons); les filles quittaient le village familial pour se marier, aux temps où l'organisation chinoise était principalement territoriale.

(느) Glose de Tcheng au Sang ta ki. Li ki, Couvreur, II, p. •202 . Cf. 1a glose de K’ong Ying -ta au même texte, p. $\bullet_{218}$, et la glose du même au Che king citée p. 3 , n.3 (ㄴ1) Dans la glose citée, K'ong Ying -ta dit :

«Le corps est d'abord mis à terre ; on espère que le souffle de vie lui reviendra ; quand il n'y a plus (d'espoir) de vie (lorsqu'on a recueilli le dernier souffle sur de l'ouate de soie), on transporte le mort sur le lit (pour faire la toilette funèbre).

(42) Cf. Fêtes et chansons anciennes, Lieux-saints.

(43) Cf. Chavannes, Dieu du sol (Annales du Musée Guimet, XXI), p. • 520 sqq •

(44) Voir dans Chavannes, 1 . c. le serment conservé par le Tsouo tchouan. Dans les idées chinoises, la Terre et la mère sont parentes, prochaines, le père et le Ciel sont majestueux, sévères. M. Chavannes semble avoir voulu nier l'antiquité de l'idée de Terre -mère, pour avoir songé trop exclusivement aux manifestations cultuelles organisées, et non pas aux croyances et aux sentiments.

$(\underline{45})$ «Nei tsö », Li ki, Couvreur, I, p. • 659 •

(46) Bull. Ec. franç. d'Ext.-Or., II, p. 352 (R. P. Cadière, Coutumes populaires de la vallée de Nguon-son).

(47) De Groot, Religious system of China, I, p. 67.

(48) Sseu-ma Tsien, Tableaux chronologiques, Chavannes, III, p. 6.

(9) $S M T$, I, 5.

(므) $\underline{S M T, I}, \cdot 333$.

(1) SMT, III, 10 (note de Tch'o u Chao-souen, interpolateur de Sseu-ma Ts'ien ).

(므) SMT, III, 3 .

$(\underline{53}) \underline{S M T, I}, \bullet_{99}$.

(54) $\underline{S M T, I}, \cdot 89 \cdot$

(도) $\underline{S M T, I}, \bullet_{210}$ •

(ㅎ6) Voir Che king, Couvreur, p. 441 : «Votre repas, c'es t du millet. »

(57) $\underline{S M T, I}, \bullet_{211}$.

(58) Chavannes, Dieu du sol (dans le T’ai Chan, Ann. du Musée Guimet, t. XXI), p. 505-6. Les souverains Tcheou sont Heou-tsi, Princes des Moissons héréditaires. Cf. SMT, I, Chavannes, ${ }^{2} 254$.

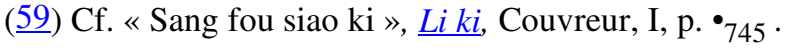

(60) Che king, «Ta ya», II, 1 (Couvreur, p. • 347 ). Tcheou song, I, 10 (Ibid., p. $\bullet_{426}$ ). Lou song, 4 (Ibid., p. ${ }_{452}$ ). Cf. Ibid., p. ${ }_{392}$. Ces hymnes, qui sont d'une grande beauté, expriment un sentiment religieux très vif et d'ordre élevé. 
(61) Che king, «Tcheou song » I, 8, Couvreur, p. ${ }_{424}$.

(62) Cette expression (formée de 4 caractères) a donné lieu à d’inutiles polé miques sur le monothéisme primitif des Chinois. Il n'y a qu'à relever dans le Che king (qui est le texte le plus ancien et le plus sûr) l'emploi des mots qui la composent pour voir que le Ciel et le Souverain ne sont qu'une même chose - et que En-haut se prend, lui aussi, dans le sens de Ciel et de Souverain. L'expression de 4 caractères n'est qu'une redondan ce poétique (de telles redondances sont fréquentes dans la titulature chinoise, religieuse ou non religieuse). Il est clair au reste, que cette expression ne traduit nullement une conception monothéiste de la divinité, et qu'elle correspond simplement à l'existence d'un culte royal adressé à une Puissance du même ordre que le pouvoir royal, universelle et absolue tout juste autant que le royaume est l' univers et le roi l'autorité unique, — non point unique, enfin, mais souveraine (au sens féodal du mot).

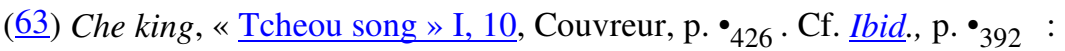

«Heou-tsi n’a plus de pouvoir ! le Souverain d'En -haut plus de bienveillance !

Comp. (Lou song, 4 , Couvreur, p. $\bullet_{454}$ ) les expressions :

«(A l') Auguste-Auguste Prince Souverain (et à l') Auguste-Ancêtre Prince (des) Moissons on offre une victime rousse.

Les titres de «Prince Souverain (d'En-Haut) » et de «Prince des Moissons » s'y balancent exactement. De même les expressions Auguste-Auguste et Auguste-Ancêtre. Auguste-Ancêtre appartient au vocabulaire du Temple Ancestral. Comp. Ibid., p. ${ }_{432}$, le balancement des expressions Auguste-Père et Auguste-Ciel. Je me bornerai à indiquer ici les principaux éléments d'une étude de ces expressions de la titulature religieuse. Le mot que je traduis par Auguste signifie brillant, lumineux, tout comme les mots employés, eux aussi, à la fois comme épithètes à Ancêtre et à Ciel. Le mot redoublé, forme un auxiliaire descriptif qui peint les fleurs brillantes (Ibid., p. ${ }_{177}$ ); même valeur (comme de juste) quand le mot n'est pas redoublé, mais précédé de la particule. Cf. Ibid., p. $\bullet_{427}$ : «quills sont brillants, l'orge et le froment !» et Ibid., p. $\bullet_{445}$ «quelle est brillante (= Auguste) cette (Maison royale des) Tcheou », ou suivi de la particule (Ibid., p. ${ }_{335}$ ) «Oh ! quil est brillant (= splendide = = Auguste) le Souverain d'En Haut!» Noter qu'ici brillant qualifie non le Ciel, mais le Souverain. Il en est de même encore dans (Cf. Ibid., p. $\bullet_{232}$ ) «qu'il a de brillant le Souverain d'En-Haut ! », même remarque). Ainsi $1^{\circ}$ les mêmes mots s'em ploient comme épithètes pour Ciel et Souverain d'En-haut; $2^{\circ}$ les mêmes pour l'Ancêtre auquel s'adresse 1e culte du temple familial ; $3^{\circ}$ ces épithètes communes à la Puissance Céleste (quel que soit le terme dont on la désigne) et à l'Ancêtre (que le culte lui associe) ont le sens concret de brillant, lumineux, splendide.

(64) Voir par exemple «Ta tchouan», Li ki, Couvreur, I, p. $\bullet_{785-7}$ et «Sang fou siao ki», Ibid., p. ${ }^{\bullet} 745-7$.

(65) Po hou t'ong. Sur la dation du nom par le Souverain, Voir Tsouo tchouan, Yin, $8^{\mathrm{e}}$ an., Legge, p. 26.

(하) $\underline{S M T, I}, \bullet_{211}$.

(67) $\underline{\text { Ibid. }} \cdot \bullet^{209} \cdot$

(68) Chouo wen.

(으) Ibid.

(70) Voir par exemple Po hou t'ong, l.c. 


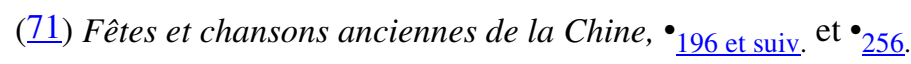

(72) Che king, «Chang song p. 3, Couvreur, p. ${ }_{462}$.

(73) Ibid., « Ta ya », II, 1, Couvreur, p. • 347 .

(74) Ibid., Lou song, 4, Couvreur, p. $\bullet 452$.

(드) Glose de Tcheng à Lou song, 4.

(76) Glose de Tcheng à Ta ya, II, 1 .

(77) Glose de Tcheng à Lou song, 4.

(78) Po hou t'ong.

(모) Chouo wen.

$(\underline{80})$ Titre de l'hymne 4 du Lou song, Couvreur, p. ${ }^{\bullet} 452$.

(81) «Yue ling », Li ki, Couvreur, I, p. ${ }_{341}$ •

(2) Et aussi de Sie (né d'un ouf d'hirondelle), autre fils du Ciel, auteur des Yin, prédécesseurs des Tcheou. Voir Fêtes et chansons, $\bullet_{164 \text { et suiv. }}$

(3) Glose de Tcheng à Lou song, 4.

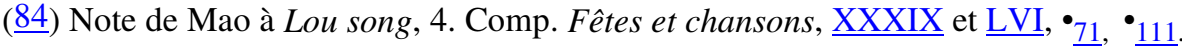

(도) Voir Yi li, notes au chapitre du «Mariage » (trad. Steele, I, p. 33) et « Houen yi », Li ki, Couvreur, II, p. ${ }_{646} \cdot$ Comp. «Wen wang che tseu », ibid., I, p. $\bullet_{483}$.

(므) Voir «Nei tsö », Li ki, Couvreur, I, p. ${ }_{662}$ (comp. « Sin chou » et « Ta tai »,. Li ki 1.c.). Voir encore Fêtes et chansons, $\underline{\mathrm{XXXIX}}$, vers 5-6, $\bullet \underline{72}$.

(7) Ta ya, II, I, Couvreur, p. • $348-9 \cdot$

(묘) Ibid.

(으) Lou song, 4 , Couvreur, p. $\bullet_{452}$.

(으) Glose de Tcheng à Ta ya, II, 1 .

(1) Idée marquée à Ta ya, II, 1. Tcheng y insiste dans sa glose.

(2) Ta ya, II, 1.

(93) Voir la préface de la première section du Che king (Kouo fong) et Fêtes et chansons, aaa et suiv., bbb et suiv. et ccc. Comp Sseu-ma Ts'ien, trad. Chavannes, I, p. 254. (Ce dernier texte indique que le principal devoir des rois était d'exercer héréditairement la charge de Prince des Moissons, Heou-tsi).

(94) Hymne 10 du Tcheou song, I, Che king, Couvreur, p. 326. On remarquera que les mots Ciel et Souverain s'appliquent à la même Puissance : celle à laquelle Heou-tsi est associé.

(95) SMT, I, 210.

(무) SMT, I, 82.

(97) Ta ya, II, I, str. 4.

(으) Ibid., str. 3 . 
(99) Cf. Si king tsa ki, chap. II (prédestination au suicide ou au parricide).

(100) SMT, I, • 281 sqq

(101) SMT, I • 209 sqq

(102) Ibid., III, 7 sqq. (note de Tch'ou chao-souen, interpolateur de Sseu-ma Ts’ien).

(103) Opinion de Mao, glose à Ta ya, II, 1.

(104) Début du livre.

(105) Sseu-ma Ts’ien et son interpolateur Tch'ou ( qui avaient consulté des versions diverses $d u$ Che king) donnent sur quelques points des indications différentes: bois bas (Che king) $=$ forêt de montagne (Sseu-ma Ts'ien) ; boufs et moutons ( Che king) = boufs et chevaux (Sseu ma Ts'ien). Sseu-ma Ts'ien, de plus, s'est efforcé de donner à la légende une couleur de vraisemblance : il dit : «boufs et chevaux ne le foulèrent point aux pieds », au lieu de «le protégèrent ».

(106) Ta ya, II, I, str. 5.

(107) SMT, I, • 210 •

(108) Comp. les jeunes fils du roi Siuan exposés sur le lit.

(109) Glose de Mao à Ta ya, II, I, str. 3.

(110) SMT, I, 210. Cf. note 55.

(111) Le Han che war tchouan, chap. I, dit :

«Un homme, à la naissance, n'est point complet à cinq points de vue ; ses yeux ne voient pas ; - il ne peut pas manger, - marcher, — parler, — engendrer. A 3 mois, il distingue et peut regarder; - à 7 mois les dents poussent et il peut manger, - à un an ses os sont devenus solides et il peut marcher; - à 3 ans les fontanelles se soudent et il peut parler ; - à 16 ans le sperme s'écoule et il peut engendrer. Le yin et le yang s'opposent : Le yang évolue sous l'action du yin : un garçon a des dents à 8 mois, et change de dents à 8 ans; à 16 ans $(8 \times 2)$ le sperme commence à s'écouler. Une fille a des dents à 7 mois et en change à 7 ans; à 14 ans $(7$ x 2) le sperme commence à s'écouler. Le yang évolue sous l'action du Pin; le yin sous l'action du yang.

D'après une théorie, en effe $t$, le nombre du petit yin est 8 , celui du petit yang 7. (Comp. « Ta tai », Li ki, chap. 80 et Chouo wen; Voir encore Kao Yeou, glose à Houai-nan tseu, chap. 23, Tchang Cheou-tsie, glose à SMT, V, p. 287 et Houang-ti nei king, chap. 1). Il s'ensuit que le retour d'âge a lieu à $7 \times 7=49=50$ ans et le terme de la virilité à $8 \times 8=64=70$ ans (Cf. $\mathrm{Li}$ $k i$, «Nei tsö »,Couvreur, I, p. $\left.{ }_{661}\right)$. Ces spéculations ont pour but de concilier les théories cosmogoniques sur le yin et le yang, certains faits d'expérience et le fait que le nombre 7 a une valeur religieuse : l'embryon est formé à 7 mois (naissance à 10 mois); - la dentition se fait. à 7 mois et à 7 ans; à 7 ans séparation des filles et des garçons, à 10 ans, le garçon sort du gynécée ; - la retraite (vieillesse) se fait à 70 ans (le terme théorique de la vie étant 100 ans). J'ai montré [dans « La vie et la mort » (Annuaire de l'Ecole des Hautes Etudes, section des sciences religieuses, 1920-1921] que 10 était pour les Chinois le nombre des durées totales; le nombre des durées totales affectées à des stages, $7(=10-3)$ la période complémentaire d'un stage. Exemple : l'enfant vit trois ans (les trois premières années) avec sa nourrice, sur les dix ans qu'il passe dans le gynécée (stage d'entrée) ; il vit trois ans séparé des filles (les trois dernières de ces dix années) sur les dix ans qu’il passe dans le gynécée (stage de sortie). Un homme (dont la vie — théoriquement — est de 100 ans) pénètre à 30 ans dans la société civile et en sort 30 ans avant la mort (à 70 ans). La période complète de coefficient 10 se décompose en deux périodes de coefficients 3 et 7 (ceci de deux façons différentes 
$10=3+(4+3)=((4+3)+3)$. D'où la valeur caractéristique du nombre 7 , le rôle qu'il jouait, et les spéculations par quoi les Chinois ont voulu expliquer cette valeur. (Voir La vie et la mort ....)

(112) Le «Nei tsö » décrit tout au long la cérémonie avec ses variantes : selon la classe sociale du père, le rang de la mère, et le fait que l'enfant est aîné ou cadet, $\mathrm{Li}$ ki, Couvreur, I, p. ${ }^{\circ} 665$ à 672 .

(113) Voir Fêtes et chansons, LXVI, v. 41.

$(\underline{114}) \lll$ Tseng tseu wen », Li ki, Couvreur, I, p. $\bullet 430 \cdot$

(115) Yi li, notes au chapitre du « Mariage », Steele, I, p. 36

(116) Tsouo tchouan Tcheng, $9^{\mathrm{e}}$ an., Legge, p. 371 Le nom de la cérémonie indique qu'alors la livraison de la fille au mari est définitive.

(117) Voir Che king, Couvreur, p. $\bullet 113$ et Granet, $\underline{\text { Coutumes matrimoniales. }}$

(118) Yi li, «Mariage », Steele, I, p. 30-31, » Kiao t’ö cheng », Li ki, Couvreur, I, p. • 610 et « Houen yi », Ibid., II, p. ${ }_{6} 646$.

$(\underline{119})$ Voir « Kiao t 'ö cheng », Li ki, Couvreur, I, p. • 661 et « Tseng tseu wen », Ibid., p. • ${ }_{429}$ •

(120) « Tseng tseu wen », Li ki, Couvreur, I, p. $\bullet_{411}$ à $\bullet_{415}$.

$(\underline{121})$ - Il y a aussi de grandes chances pour que certaines interdictions caractéristiques des trois premiers jours du mariage aient été étendues aux 3 premiers mois dans les hautes classes de la noblesse (Voir Coutumes matrimoniales, Appendice. La dation du nom qui, dans la famille organisée agnatiquement, ne se fait qu'au $3^{\mathrm{e}}$ mois (par l'autorité du père) a dû d'abord se faire le $3^{\mathrm{e}}$ jour (et sans doute par la mère : tel semble être le cas pour Heou tsi).

(122) Voir « Ta tai », Li ki, chap. 48 et Sin chou, $l c$.

(123) Le deuil est abaissé d'une classe : simplement pour montrer que, le mariage fait, l'autorité suprême sur la femme appartient au mari. Yi li, « Deuil », Steele, II, p. 20.

$(\underline{124})$ «Tsa ki », Li ki, Couvreur, II, p. • 197 : formes de la répudiation.

$(\underline{125})$ «Ta tai », Li ki, chap. 80. Il y a 3 cas où la répudiation est interdite. $1^{\circ}$ quand (la femme) a eu (une famille où on l'a prise) et qu'elle n'a plus (de famille) où revenir $=; 2^{\circ}$ quand elle a aidé à porter le deuil des parents du mari ; $3^{\circ}$ quand, s'étant mar ié pauvre, (le mari) est par la suite devenu riche.

$(\underline{126})$ «Nei tsö », Li ki, Couvreur, I, p. • ${ }_{674}$ et p. $\bullet \underline{625}$.

$(\underline{127})$ «Kouan yi », Li ki Couvreur, II, p. • 637 sqq $•$

$(\underline{128})$ « Nei tsö », Li ki, Couvreur, I, p. • 624 .

(129) Kouan $Y i, 1$. c. et $Y i$ li «Majorité », Steele, I, p. 9. Cette visite marque sa sortie définitive du gynécée : il prend congé de sa mère.

(130) Voir Fêtes et chansons, p. • $157 \mathrm{sqq} \cdot$ et p. • $169 \cdot$

$(\underline{131})$ « Nei tsö », Li ki, Couvreur, I, p. • 673 • «Wang tche », Ibid., p. • 301 et «Wen wang tse tseu », Ibid., p. • 470-472. Voir SMT, IV, p. 29, p. 46, p. 63, et principalement p. 59. Le rôle de l'oncle maternel, peu observable dans les textes anciens, est d'une importance marquée dans les usages modernes. Cf. la coutume actuelle des marchands qui font faire à leur fils leur apprentissage chez un confrère, et des lettrés qui confient à un autre lettré, l'éducation de leurs enfants. 
(132) Les aliments sucrés, tendres, succulents. «Nei tsö », Li ki, Couvreur I, p. • ${ }_{627}$ et p.

- 655 sqq - De même, enfants et vieillards échappent aux châtiments du droit pénal : « K’iu li ., Ibid., p. 9.

$(\underline{133})$ « Nei tsö », Li ki, Couvreur, I, p. $\bullet 661$.

(134) Ibid., p. • 673 .

$(\underline{135})$ La mère plébéienne nourrit l'enfant dès le $3^{\mathrm{e}}$ jour : elle est sa nourrice ; mais le rapport de mère à fils n'est établi qu'au $3{ }^{\mathrm{e}}$ mois, quand, à la cérémonie des relevailles, le père remet l'enfant à la mère pour qu'elle l'élève.

(136) Tels sont les 3 premiers signes prémonitoires du pouvoir paternel et du caractère viril de l'enfant : rites d'approche.

$(137)$ « T’an Kong », Li ki, Couvreur, I, p. • 118 •

(138) Voir les références de la note 111.

(139) Роио ya, chap. 6.

$(\underline{140})$ Voir au $\mathrm{Li} k i$ «Nei tsö », Couvreur, p. $\bullet{ }_{649}$, les préparatifs funéraires faits pour les vieillards à partir de 60 et surtout de 70 ans. Ibid., p. $\bullet{ }_{651}$, à 70 ans on quitte la vie publique : Voir une explication de la retraite à 70 ans dans le «Tseng tseu wen», Li ki, Couvreur, I, p. $\bullet 418$. Comp. «Wang tche », Ibid., p. $\bullet_{\underline{315}}$ et p• $\underline{320}$. «Kiu li », Ibid., p. • 9 . La vie a son terme théorique à 100 ans ; à 70 ans commence la retraite qui, théoriquement, doit durer 30 ans.

(141) Les cérémonies du début de la mort sont décrites dans le Yi li, chap. XXVI, Steele, II, p. 45 sqq. et dans le «Sang ta ki », Li ki, Couvreur, II, p. • 202 sqq. Voir encore «T’an kong », Ibid., I, p. $\bullet 199$ sqq.

$(\underline{142})$ « T’an kong », Li ki, Couvreur, I, p. • 204 , glose de K'ong Yng -ta.

$(\underline{143})$ Sur les rites des $3^{\mathrm{e}}$ jour, $3^{\mathrm{e}}$ mois, $3^{\mathrm{e}}$ année, voir «T’an kong », Ibid., p. $\bullet 116$ (comp. Ibid., p. $\bullet{ }_{127}$ et p. $\bullet{ }_{142}$ ). La sortie de la maison est bien marquée par le même traité (Ibid., p. 150) : progression à la fois continue et rythmée. Le coefficient des premières périodes du deuil peut varier en fonction de la dignité du défunt: Cf. «Wan tche», Ibid., p. • 286 . La durée du grand deuil ( 3 ans) est constante. On attribue à Confucius une assimilation des 3 années du deuil aux 3 années que dure la nourriture de l'enfant : «San nien wen », Ibid., II, p. - 586 . Dans ce dernier traité se voit lindication de la valeur totale des différentes périodes de deuil, p. $\bullet 584-5$.

(144) Sur les tablettes provisoires et définitives, sur l'association aux offrandes faites au grand-père et l'assignation d'une place spéciale, Voir aussi le «T’an kong »ibid, p. • 201 et - 205 sqq . Comp «Fang ki », Ibid., II, p. • 413 .

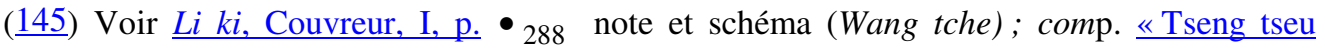
wen », Ibid., p. $\bullet 435$. (Noter l'usage de réunir pendant le deuil les tablettes des ancêtres autour de celle du plus ancien auteur de la famille: de même tous les parents se groupent autour du chef du deuil.). «Sang fou siao ki », Ibid., p. ${ }_{746}$ et «Ta tchouan », Ibid., p. $\bullet 785$.

(146) Sur le tabou du nom des parents défunts, voir «K’iu li », Li ki, Couvreur, I, p. $\bullet 56$ sqq. «Tsa ki », Ibid., II, p. $\bullet{ }_{179}$ et p. $\bullet \underline{193}$. Sur la levée du tabou correspondante, Voir «T’an 
kong», ibid, I, p. $\bullet_{241}$. Noter l'usage de la clochette à battant de bois. Rap. «Yue ling », $\underline{\text { Ibid., p. }} \bullet{ }_{342}$ : Voir Granet, Coutumes matrimoniales, note 74.

(147) L'emploi d'un nombre défini de noms personnels pour une famill e déterminée n'est, en aucune façon, attesté pour l'ancienne Chine. De nos jours, souvent le premier des deux caractères formant ce nom est commun à tous les membres d'une même génération et choisi dans une devise, adoptée par la famille, qui semble être d'ordinaire composée de 5 caractères ou de 4 ou d'un multiple de 4 . Cette méthode devrait être étudiée de près.

$(\underline{148})$ «T'an kong », Li ki, Couvreur, I, p. $\bullet 205$ et p. • 241 . «Kiu li », Ibid., p. • 47 • «Tseng tseu wen », ibid.., p. 455. «Tsi t'ong » ibid., II, p. $\bullet 336$. Voir encore «Fang ki », ibid., II, p. 413 et « Kiao t 'o cheng, ibid :, I, p. • 613 .

$(\underline{149})$ «Tsi t'ong », Li ki, Couvreur, II, p. • 335 .

(150) Yi li, « Deuil », Steele, II, p. 11.

(151) Voir plus haut.

(152) Koио yu, « Tcheou yu » $1^{\mathrm{e}}$ Partie.

(153) Fêtes et chansons, p. $\bullet 200$ sqq

(154) Tsouo tchouan. Tchao, $7^{\mathrm{e}}$ an. Legge, p. 618 (comp. la traduction du P. WIEGER, Textes philosophiques, p. 110). Le sage ministre Tseu-tch'an, questionné sur la survie, répond :

«Quand un homme est engendré, au début de l'évolution (embryonnaire) il y a ce qu'on appelle le p'o. Quand est né (ce) p'o, (la partie) yang (de ce p'o) s'appelle le houen.

Les commentaires de $K^{\prime}$ 'ong Ying-ta font du p'o l'âme sensitive, du houen l'âme intelligente. $\mathrm{Ne}$ retenons que l'essentiel du texte savoir que la formation d'une personnalité humaine se fait en deux temps, le houen n'apparaissant qu'après le p'o.

$(\underline{155})$ « Kiu li », Li Ki, Couvreur, I, p. • 85 [note 104] · «T'an kong », ibid., p. $\bullet{ }_{199-200} \cdot$ « Li yun », ibid., p. $\bullet 503$. «Sang ta ki », ibid., p. $\bullet_{204 \text { sqq }}$.

$(\underline{156})$ «T’an kong », Li ki, Couvreur, I, p. • 246 . Confucius admira la science du cérémonial montrée par le héros de cette anecdote. Comp. «Kiao t’o cheng », Ibid., p. • ${ }_{612-3}$. Au p'o semblent s'adresser les libations qui transportent l'effet du sacrifice jusqu'aux sources souterraines; au houen, les exhalaisons (graisse brûlée avec de l'armoise) qui transportent l'effet du sacrifice au del à du toit, jusqu'au ciel.

(157) La criée, le recueillement du souffle et le rappel du houen sont des procédés de mise en demeure. Par la façon dont il se conduit, quand ils sont employés, celui pour qui l'on procède se prouve, ou non, mort ; on lui donne les moyens de la preuve et il est contraint de la faire : s'il ne reconstitue pas sa personnalité, elle doit se dissoudre.

(158) L'affiliation n'est complète qu'à la majorité.

(159) Voir plus haut le texte de Sseu-ma Ts’ien.

(160) Chou king, chap. "Yi-tsi ». Yu, pour montrer quil est homme de devoir, rappelle par des traits essentiels son mariage et la naissance de son fils, événements auxquels il ne consacra que le moins de temps possible. Reproduire l'onomatopée caractéristique des vagissements lui suffit pour exprimer que, la naissance sitôt acquise, il est parti travailler aux canaux de la Chine. 
(161) Idée qui a persisté ; Voir Wieger, Folklore chinois, p. • ${ }_{79}$ (Voir aussi p. 65 et p. 66 et, pour un exemple de réincarnation dans la famille p. $\bullet \underline{334 \mathrm{sqq}} \cdot$ ).

(162) Voir «T'an kong », Li ki, Couvreur, I, p. $\bullet 131$. Noter le proverbe : « Le renard mourant tourne la tête vers la colline natale. »

(163) Idée du pouvoir régulateur qu'un chef doit posséder à la fois sur son peuple et sur la Terre, sur les hommes et sur la Nature. Voir Granet, Fêtes et chansons, p. 202 et p. 250.

(164) Voir Ibid., p. 130, n. 6, p. 156 et p. 238. La comparaison des légendes relatives aux naissances du Dieu agraire Heou-tsi et du sage Confucius montre bien le passage de l'idée de Lieu Saint à celle, plus définie, de Centre Ancestral. Heou-tsi eut la stature et la fermeté d'un géant : il était né du pas d'un géant. Confucius avait le crâne en forme de colline Kieou : sa mère le conçut en priant sur la colline $K^{\prime}$ 'ieou $\mathrm{Ni}$; il porta les noms de $\mathrm{K}^{\prime}$ ieou et de $\mathrm{Ni}$ (cf. ibid., p. 130, n. 6 et Sseu-ma Ts'ien éd. Chavannes, V, p. 288 sqq.). Heou-tsi était né pour être le Prince des Moissons d'un pays : sa mère le conçut par des pratiques analogues à celles qu'employaient les filles du pays de Tcheng, par exemple, quand elles obtenaient des réincarnations aux fêtes du Lieu Saint de Tcheng où toutes les familles du pays se trouvaient réunies. La naissance de Confucius avait été désirée pour fournir un chef de culte (son père ayant déjà un fils, mais infirme et impropre aux choses sacrées) à la famille $K^{\prime}$ ong : sa mère le conçut en allant prier sur la colline où était le Temple Ancestral de la famille K'ong.

(165) Voir plus haut.

$(\underline{166})$ « Tseng tseu wen », Li ki, Couvreur, I, p. 460.

$(\underline{167})$ «T'an kong », Li ki, Couvreur, I, p. • 157 .

(168) Sur la cabane de deuil, Voir «Sang ta ki », Li ki. Couvreur, II, p. 239 sqq. ; « Tsa ki », Ibid., p. 120 et p. 163 ; «Fou wen», Ibid., p. 555 ; «Kien tchouan», Ibid., p. 573 sqq. et p. 581.

(169) Comp. l'usage Lolo. Le cadavre est enterré provisoirement au côté de la maison et s'y décompose; les parents surveillent attentivement cette décomposition. Quand est fait l'e nterrement définitif, on procède à une cérémonie pour réunir 1'àme du nouvel Ancêtre à celles des Ancêtres de la même génération: auparavant cette âme demeurait isolée dans un coin de la maison. Voir BONIFACY, B. E. F. E. O., VIII, p. 548, p. 552 et p. 541.

(170) Les parents morts avant leur majorité ne reçoivent point d'offrandes dans ce coin sacré et n'ont pas, non plus, le droit d'avoir un représentant : ils n'ont point engendré. Cf. «Tseng tseu wen », Li ki, Couvreur, I, p. 457 et p. 455.

(171) On trouvera quelques éléments d'une étude des représentations chi noises anciennes relatives à la survie dans Granet, La vie et la mort, Annuaire de I Ecole des Hautes Etudes, section des Sciences religieuses, 1920-1921. Les libations du culte funéraire sont dites atteindre les sources profondes, c'est-à-dire les Sources jaunes, situées sous terre, non loin $d u$ pays natal, vers le nord. De ces sources semblent s'échapper en même temps (au printemps) l'eau des fontaines qui jaillit après avoir été tarie l'hiver et les âmes que l'on peut faire se réincarner à l'aide de rites pratiqués sur l'eau des fontaines. Des Sources jaunes, séjour des morts, vient aussi le Tien-kouei, principe des sécrétions fécondes de l'homme et de la femme.

(172) Formule poétique consacrée. Cf. Che king, «Tcheou song», III, 5 et 6, Couvreur, p. 439 et 441 .

(173) Voir le texte du Tcheou ti et la glose de Tcheng dans : Coutumes matrimoniales, supra, p. $84, n .3$. 
(174) Chouen accède à l'Empire comme gendre de Yao (SSEU-MA-TS'IEN, Chavannes, I, p. 73). Ts'in Che-houang-ti lutte contre la coutume des maris-gendres (ibid. II, p. 168). (En Annam, le code des Le cherche encore à linterdire : B. E. F. E.- O., X, p. 35.) L'usage s'en est maintenu jusqu'à nos jours. Voir P. HOANG, Mariage chinois, p. 99 sqq. (avec quelques exemples historiques).

$(\underline{175})$ La vraie maison c'est le gynécée.

(176) Voir plus haut.

(177) Voir plus haut, les cas du roi Mou et celui du comte Lan.

(178) M. Chavannes (Dieu du sol, l.c.) a, selon mon gré, mis trop uniquement l'accent sur les représentations masculines données au Sol sur lequel s'exerce le pouvoir seigneurial.

(179) Au vassal chargé de porter l'enfant correspond u ne gardienne ; la mère ne prend qu'exceptionnellement l'enfant dans les bras ; le père ne peut l'y porter (le grand-père le peut : grand-père et petit-fils agnatiques sont déjà parents en droit utérin). 
$\underline{\text { I. }} \quad \underline{\text { II. }=} \quad \underline{\text { III. }-} \quad \underline{\text { Notes }}: \underline{1}-\underline{50}-\underline{100}-\underline{150}$ 
Nom du document : depot_enfant.doc

Dossier :

C:ICSSIEnvoi021204/granet_marcel

Modèle :

C:IWINDOWS $\backslash$ Application

DatalMicrosoftlModèles\Normal.dot

Titre :

Sujet :

Auteur :

Le dépôt de l' enfant sur le sol

Mots clés :

série Chine

Marcel Granet

chinoise, Chine ancienne, Chine antique, Chine classique, ethnologie de la Chine, mythologie chinoise, sociologie de la Chine, confucius, civilisation chinois, religion chinoise, ancient China, sinol

Commentaires : iences_sociales/index.html

http://www.uqac.uquebec.ca/zone30/Classiques_des_sc

Date de création : $\quad$ 13/11/04 13:17

$\mathrm{N}^{\circ}$ de révision : $\quad 6$

Dernier enregistr. le : 01/12/04 21:55

Dernier enregistrement par : Pierre Palpant

Temps total d' édition12 Minutes

Dernière impression sur : $\quad 05 / 12 / 04$ 12:15

Tel qu' à la dernière impression

Nombre de pages :

46

Nombre de mots : 20038 (approx.)

Nombre de caractères : 114217 (approx.) 